\title{
Graded consequence: an institution theoretic study
}

\author{
Răzvan Diaconescu \\ Simion Stoilow Institute of Mathematics of the Romanian Academy
}

\begin{abstract}
We develop a general study of graded consequence (of many-valued logic) in an institution theoretic (in the sense of Goguen and Burstall) style. This means both syntax and semantics are considered fully abstract, as well as the satisfaction between them. Our approach contrasts to other approaches on many-valued logic in that it is a multisignature one, in the spirit of institution theory. We consider graded consequence at three different conceptual levels: entailment, semantic, and closure operators, and explore several interpretations between them. We also study logical connectors and quantifiers both at the entailment and semantic level, compactness and soundness properties.
\end{abstract}

\section{Introduction}

Generally speaking, mainstream many-valued logic (e.g. [25]) has a crisp approach to consequence. However there have also been a significant interest in graded approaches to consequence, in which the logical derivations need not be either true or false. Mathematical theories on graded consequences add a new dimension to many-valued logic and constitute an important conceptual tool for approximate reasoning. Non-crisp consequence has been considered implicitly in the rather early work [34], and more explicitly later on by other works such as [4, 5, 16, 20], etc.

Our general and foundational study of the concept of graded consequence is done in the style of the institution theory of Goguen and Burstall [23] at three different levels: (1) entailment, (2) semantic, and (3) in terms of closures. Institution theory, understood here more in its wider mathematical cultural meaning then in its strictly technical meaning, is a categorical abstract model theory that arose about three decades ago [22] as a response to the explosion of the population of logical systems used for formal software specification. Its original aim was to develop as much computing science as possible in a general, uniform way, independently of particular logical systems. This has been achieved to an extent even greater than originally envisaged. The theory of institutions became the most fundamental mathematical tool underlying algebraic specification theory (in its wider meaning) [36], also being increasingly used in other areas of computer science. Moreover, institution theory is a major trend in the so-called 'universal logic' (in the sense envisaged by Jean-Yves Béziau [1,2]) which is considered by many a true renaissance of mathematical logic. A lot of model theory has been gradually developed at the level of abstract institutions (see [10]). A relatively recent survey of the vast area of institution theory is [13].

Our approach to graded consequence is a multi-signature one in the spirit and tradition of institution theory, a distinctive aspect which contrasts with the other abstract approaches to graded consequence (e.g. [4, 34]). This means that we explicitly consider translations between signatures together with the induced corresponding translations both at the level of the syntax (sentences) and of the semantics (models). Multi-signature frameworks are absolutely indispensable both from the side of engineering and of logic. On the one hand, foundations of module systems both in specification and programming was one of the original main motivations for institution theory [23] and these rely crucially upon the multi-signature aspect of institutions. On the other hand, in order to address at the abstract level several important logic concepts such as quantifiers, interpolation, definability, etc. one needs an explicit multisignature framework (see [10, 32]). Moreover the abstract view of several model theoretic methods, such as diagrams, saturated models, etc. [10], also require an explicit signature morphisms as primitive concept.

Email address: Razvan. Diaconescu@imar.ro (Răzvan Diaconescu) 


\section{Summary and Contributions.}

1. We start by recalling a series of order theoretic concepts necessary for our work.

2. Then we introduce our entailment-styled concept of graded consequence which may be regarded either as an extension of the main definition of [4] to a multi-signature context or as a generalisation of classical two-valued entailment systems from institution theory [30] to many-valued truth. We also discuss 'graded proofs', which are entailments of the graded entailment systems freely generated by systems of raw graded rules.

3. The semantic level of our study of graded consequence is based upon a natural generalization of the concept of institution from the classical two-valued to the many-valued situation. Like in the case of classical institutions, many-valued institutions also give rise to Galois connections between the syntax and the semantics. We interpret many-valued institutions as graded entailment systems by defining a corresponding graded semantic consequence; this generalizes the ordinary semantic consequence of classical institutions. Moreover we show that this is a retract, its inverse being a construction of an artificial many-valued institution that provides a technical artefact supporting semantic arguments in abstract consequence theoretic contexts. The conceptual mixture between graded entailment systems and many-valued institutions provides a fully abstract setting for discussing soundness in a many-valued context. In this context we show that the soundness property may be automatically transferred from rules to graded proofs.

4. The many-valued closure systems, representing the third level of our study of graded consequence, may be regarded either as an extension of Pavelka's consequence operators [34] to multi-signature contexts or as a generalization of $\pi$-institutions of [18] to many-valued. Unlike in the classical two-valued case where entailment systems and closure systems are one concept, in the many-valued case the relationship between these two concepts is far more complex. We present two interpretations of graded entailment systems as many-valued closure systems. One owes inspiration to Goguen's many-valued interpretation of Modus Ponens [21], while the second one corresponds to a semantic closure. Although in the classical two-valued situation both of them collapse to the ordinary closure under entailment, in the proper many-valued situations they may be different. We show that in general the former closure is weaker than the latter. The opposite move, from closure systems to graded entailment systems is however more problematic, especially in a proper multi-signature context.

5. Next we study the 'logic of graded consequence' first by introducing logical connectors and quantifiers at two different levels, proof theoretic (i.e. that of entailments) and semantic (i.e. that of institutions), and then by providing sufficient conditions such that the presence of these at the latter level induces their presence at the former level (i.e. the level of graded semantic consequence). Here we also show that the soundness property is preserved by logical connectors (with the exception of disjunction $\vee$ ) and quantifiers.

6. In the last technical section we propose a natural generalisation of compactness for entailment systems from classical two-valued to many-valued truth. We show that finitary rules generate compact entailments and that compactness is preserved by logical connectors and quantifiers.

7. At the conclusions we compile a list a open question that arise from our study.

Because of its institution theory side, our paper involves some very elementary category theory, up to the concept of natural transformation. With few exceptions, in general we follow the terminology and the notations of [28]. As regards the notational conventions, $|C|$ denotes the class of objects of a category $C, C(A, B)$ the set of arrows (morphisms) with domain $A$ and codomain $B, f ; g$ the composition in diagrammatic order which reads as $f \circ g$, the composition in set theoretic order. The category of sets (as objects) and functions (as arrows) is denoted by Set, and the category of all categories (as objects) and functors (as arrows) is denoted by CAT. Strictly speaking, CAT is only a 'quasi-category' living in a higher set-theoretic universe.

\section{Order theoretic preliminaries}

This section is devoted to recalling of several order theoretic concepts and results that we are going to use in our work. For general lattice theory we recommend [24], and for residuated lattices [19].

An integral commutative residuated lattice $\mathcal{L}=(L, \leq, \wedge, \vee, \top, \perp, *, \Rightarrow)$ is a bounded lattice (with $\leq$ denoting the underlying partial order that has infimum $\wedge$, supremum $\vee$, biggest $T$ and lowest $\perp$ elements) and which comes equipped with an additional commutative and associative binary operation $*$ which has $T$ as identity and such that for all elements $x, y$ and $z$ 
$-(x * y) \leq(x * z)$ if $y \leq z$, and

$-y \leq(x \Rightarrow z)$ if and only if $x * y \leq z$.

The first condition just means that $(x *-)$ is a functor on the partial order $(L, \leq)$, and the second condition means that it has a right adjoint $(x \Rightarrow-)$. The ordinary two-valued situation can be recovered when $\mathcal{L}$ is the two values Boolean algebra with $*$ being the conjunction. Then $\Rightarrow$ is the ordinary Boolean implication. In general $x * y \leq x \wedge y$; when $x * y=x \wedge y, \mathcal{L}$ is called a Heyting algebra.

In what follows we will call integral commutative residuated lattices just 'residuated lattices'. There is a myriad of interesting examples of residuated lattices used for many-valued logics for which $*$ gets an interpretation rather different from the ordinary conjunction. One famous such example is the so-called Eukasiewicz arithmetic conjunction on the closed interval $[0,1]$ or on the sets $\left\{0, \frac{1}{n}, \frac{2}{n}, \ldots, \frac{n-1}{n}, 1\right\}$ defined by $x * y=1-\min \{1,2-(x+y)\}$. In this examples $x \Rightarrow y=\min \{1,1-x+y\}$.

In a partially ordered set $(L, \leq)$ an element $x$ is called compact (or finite) if for every directed subset $D$ of $L$, if $D$ has a supremum $\vee D$ and $x \leq \bigvee D$ then $x \leq d$ for some $d \in D$. $(L, \leq)$ is compact when all its elements are compact. Any partially ordered set is trivially compact. The totally ordered set $\{0\} \cup\left\{\frac{1}{n} \mid n \in \omega\right\}$ is an example of an infinite compact partial order; it is also a complete Heyting algebra.

In any complete lattice $\mathcal{L}$ a function $f: L \rightarrow L$ is meet-continuous when for any non-empty family $\left(x_{i}\right)_{i \in I}$, $f\left(\bigwedge_{i} x_{i}\right)=\bigwedge_{i} f\left(x_{i}\right)$, it is join-continouus when $f\left(\bigvee_{i} x_{i}\right)=\bigvee_{i} f\left(x_{i}\right)$, and it is continuous when it is both meet- and join-continuous. Note that any meet- or join-continuous function $f$ is increasing monotonic, i.e. $x \leq y$ implies $f(x) \leq f(y)$. A completely distributive lattice is a complete lattice in which arbitrary joins distribute over arbitrary meets.

In any partial order $(L, \leq)$ a closure operator is an increasing monotonic function $C: L \rightarrow L$ such that for each $x \in L, x \leq C(x)$ and such that $C \circ C=C$.

A Galois connection consists of a pair of increasing monotonic functions between two partial orders, $f:(P, \leq) \rightarrow$ $(Q, \leq)$ and $g:(Q, \leq) \rightarrow(P, \leq)$ that form an adjunction when $f, g$ are regarded as functors (in the sense of category theory [28]).

The results stated by Prop. 2.1, 2.2, 2.3 below, that will be used in what follows, are well known in the lattice theory literature (e.g. [33]); in fact they are easy to establish by the reader herself. Hence we give them without proof.

Proposition 2.1. The following relations hold in any residuated lattice:

$$
\begin{gathered}
(x \Rightarrow x)=\top \\
x^{\prime} \leq x \text { implies }(x \Rightarrow y) \leq\left(x^{\prime} \Rightarrow y\right) . \\
\bigwedge_{i} x_{i} \leq \bigwedge_{i} y_{i} \text { when } x_{i} \leq y_{i} \text { for each } i \in I . \\
\bigwedge_{i}\left(x \Rightarrow y_{i}\right)=\left(x \Rightarrow \bigwedge_{i} y_{i}\right) \\
(x \Rightarrow y) *(y \Rightarrow z) \leq(x \Rightarrow z) \\
\left(\bigvee_{i} x_{i}\right) * y=\bigvee_{i}\left(x_{i} * y\right) \\
(\perp \Rightarrow x)=\top \\
(\top \Rightarrow x)=x
\end{gathered}
$$

Proposition 2.2. The following relations hold in any Heyting algebra:

$$
\begin{gathered}
((x \wedge y) \Rightarrow z)=(x \Rightarrow(y \Rightarrow z)) . \\
x \wedge(x \Rightarrow \perp)=\perp .
\end{gathered}
$$

Proposition 2.3. The following relation holds in any Boolean algebra:

$$
x \Rightarrow(y \vee z)=(x \Rightarrow y) \vee(x \Rightarrow z) \text {. }
$$




\section{3. $\mathcal{L}$-entailment}

The structure of this section is as follows.

1. We first introduce the concept of systems of graded rules that represent raw entailments.

2. Then we introduce the concept of graded entailment systems by imposing a set of axioms on systems of graded rule.

3. In the last part of the section we introduce the concept of graded proofs as graded entailments that are generated by graded rules.

\subsection{Graded rules}

The concepts introduced in this paper are developed over a fully abstract syntax, in the style and tradition of institution theory. This means that signatures are considered explicit but as objects in abstract categories, with the arrows or morphisms standing for the translations between the signatures. To each signature we relate the set of its sentences, also considered fully abstract. Any signature morphism determines a translation between the corresponding sets of sentences, and this is functorial. These ideas are formally captured by the following definition that borrows terminology from [35]:

Definition 3.1 (Logic syntax). A logic syntax is a pair (Sign, Sen) such that

1. Sign is a category whose objects are called 'signatures' and whose arrows are called 'signature morphisms', and

2. a functor Sen : Sign $\rightarrow$ Set called 'sentence functor'; the objects of $\operatorname{Sen}(\Sigma)$ are called ' $\Sigma$-sentences'.

In what follows we may abbreviate $\operatorname{Sen}(\varphi)(\rho)$ by $\varphi(\rho)$.

The essence of the multi-signature aspect of this definition refers more to having proper signature morphisms rather than having several signatures. Indeed, [40] discusses an abstract framework that has only one signature and in which substitutions are assimilated to signature morphisms.

Definition 3.2 (Many-valued theories). For any fixed set $L$ and for any logic syntax (Sign, $\operatorname{Sen})$, an $\Sigma$-( $L$ )-theory is just a function $X: \operatorname{Sen}(\Sigma) \rightarrow L$.

In the fuzzy sets literature terminology $\Sigma$-theories are just $L$-sets, our definition generalizing concrete concepts of fuzzy theories (e.g. [6]). Note that the classical two-valued concept of theory as a set of sentences for a given fixed signature is obviously subsumed by Dfn. 3.2 since a function $\operatorname{Sen}(\Sigma) \rightarrow 2$ is the same with a subset of $\operatorname{Sen}(\Sigma)$. In this case $X(\rho)=1$ means $\rho \in X$.

Notation 3.1. When $\mathcal{L}=(L, \leq, \wedge)$ is a complete meet-semilattice, for any $L$-set $X: S \rightarrow L$ and for any $E \subseteq S$ we denote

$$
X(E)=\bigwedge\{X(e) \mid e \in E\} .
$$

Definition 3.3 ( $L$-rules). Let $L$ be any set. A tuple (Sign, Sen, $\left.\left(\vdash_{\Sigma}\right)_{\Sigma \in|\operatorname{Sign}|}\right)$ is an $L$-rules system when

- (Sign, Sen) is a logic syntax, and

$-r_{\Sigma}$ is an L-valued relation $r_{\Sigma}: \mathcal{P}(\operatorname{Sen}(\Sigma)) \times \operatorname{Sen}(\Sigma) \rightarrow L$.

When $\mathcal{L}=(L, \leq)$ is a partial order an $\mathcal{L}$-rules system means an L-rules system. When the signature is clear from the context we may skip the subscript from $r_{\Sigma}$ and simply write + instead. Also when the logic syntax is clear from the context we may abbreviate (Sign, Sen, $\left.\left(\vdash_{\Sigma}\right)_{\Sigma \in \mid \text { Sign } \mid}\right)$ by $\vdash$ only.

When $\mathcal{L}$ is partial order with a lowest element $\perp$, we say that the $\mathcal{L}$-rules system is finitary if $E \vdash \gamma=\perp$ whenever $E$ is infinite. 
Example 3.1. Let the objects of Sign be families of sets $\Sigma=\left(\Sigma_{n}\right)_{n \in \omega}$, with $\Sigma_{n}$ representing operation symbols of arity $n$. A morphism $\varphi: \Sigma \rightarrow \Sigma^{\prime}$ consists of a family of functions $\varphi=\left(\varphi_{n}\right)_{n \in \omega}$ where $\varphi_{n}: \Sigma_{n} \rightarrow \Sigma_{n}^{\prime}$. For each $\Sigma$, Sen $(\Sigma)$ is the set of the sentences of the form $(\forall X) t>t^{\prime}$ with $X$ being a finite block of variables and $t, t^{\prime}$ being $\Sigma(X)$-terms, i.e. terms of the signature that adds the variables $X$ as new constants to $\Sigma$.

Now let $\mathcal{L}$ be the space of truth values for the Łukasiewicz $(n+1)$-valued logic, i.e. the lattice $\left\{0, \frac{1}{n}, \frac{2}{n}, \ldots, \frac{n-1}{n}, 1\right\}$ with the residuated structure given by Łukasiewicz's arithmetic conjunction. We may define the following finitary system of $\mathcal{L}$-rules:

$$
\begin{aligned}
& \left.\begin{array}{llll}
R: & & \vdash_{\Sigma}(\forall x) x>x & =1 \\
T: & \left\{t>t^{\prime}, t^{\prime}>t^{\prime \prime}\right\} & \vdash_{\Sigma} t>t^{\prime \prime} & =1
\end{array} \quad \text { (for all } \Sigma \text {-terms } t, t^{\prime}, t^{\prime \prime}\right) \\
& S: \quad(\forall X) t>t^{\prime} \quad \vdash_{\Sigma} \theta[t]>\theta\left[t^{\prime}\right] \quad=\frac{2}{n} \quad \text { (for all } \Sigma \text {-terms } t, t^{\prime} \text { and substitution } \theta \text { ) } \\
& \sigma:\left\{t_{1}>t_{1}^{\prime}, \ldots, t_{k}>t_{k}^{\prime}\right\} \quad \vdash_{\Sigma} \sigma\left(t_{1}, \ldots, t_{k}\right)>\sigma\left(t_{1}^{\prime}, \ldots, t_{k}^{\prime}\right)=\frac{n-1}{n} \quad \text { (for all } \Sigma \text {-terms } t_{i}, t_{i}^{\prime} \text { and } \sigma \in \Sigma_{k} \text { ) }
\end{aligned}
$$

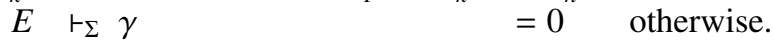

Example 3.2. Let Sign consists of only one object $\Sigma=\{p, q\}$. Let $\operatorname{Sen}(\Sigma)$ consists of the terms formed over the constants $p, q$ by the unary operations $\neg, \square$ and the binary operations $\wedge, \vee, \Rightarrow$. We take $\mathcal{L}$ to be the interval $[0,1]$ with the residuated lattice structure given by the Łukasiewicz's arithmetic conjunction. We may define the following finitary system of $\mathcal{L}$-rules:

$$
\begin{array}{rlll}
M P: \quad\{q, q \Rightarrow p\} & \vdash_{\Sigma} p & =0.9 \\
B: & \vdash_{\Sigma} \neg \square p & =0.8 \\
E & \vdash_{\Sigma} \gamma & =0 \quad \text { otherwise. }
\end{array}
$$

The following is an important notation.

Notation 3.2. Let $\mathcal{L}=(L, \leq, \wedge)$ be a complete meet-semilattice. For any $\mathcal{L}$-rules system and any sets $E, \Gamma$ of $\Sigma$ sentences we let

$$
(E \vdash \Gamma)=\bigwedge\{E \vdash \gamma \mid \gamma \in \Gamma\}
$$

Fact 3.1. Let $\mathcal{L}=(L, \leq, \wedge)$ be a complete meet-semilattice. For any $\mathcal{L}$-rules system and any sets $E, \Gamma, \Gamma^{\prime}$ of $\Sigma$ sentences we have that

$$
\left(E \vdash \Gamma \cup \Gamma^{\prime}\right)=(E \vdash \Gamma) \wedge\left(E \vdash \Gamma^{\prime}\right) .
$$

Note that in approaches that consider entailments as binary relations of $\mathcal{P}(\operatorname{Sen}(\Sigma))$ rather than relations between sets of sentences and single sentences (such as $[10,16]$ ), the equation of Fact 3.1 has to be provided as an axiom.

\subsection{Graded entailment}

Definition 3.4 ( $\mathcal{L}$-entailment). Let $\mathcal{L}=(L, \leq, *)$ such that $(L, \leq)$ is a complete meet-semilattice (with $\mathrm{T}$ denoting its upper bound) and $*$ is a binary operation on L. An $\mathcal{L}$-rules system (Sign, Sen, $\left.\left(\vdash_{\Sigma}\right)_{\Sigma \in|\operatorname{Sign}|}\right)$ is an $\mathcal{L}$-entailment system when the following axioms hold:

1. (Reflexivity) $\{\gamma\} \vdash_{\Sigma} \gamma=\mathrm{T}$.

2. (Monotonicity) $\left(E \vdash_{\Sigma} \gamma\right) \leq\left(E^{\prime} \vdash_{\Sigma} \gamma\right)$ when $E \subseteq E^{\prime}$.

3. (Transitivity) $\left(E r_{\Sigma} \Gamma\right) *\left(\Gamma r_{\Sigma} \rho\right) \leq\left(E r_{\Sigma} \rho\right)$.

4. (Translation) $\left(E \vdash_{\Sigma} \gamma\right) \leq\left(\varphi(E) \vdash_{\Sigma^{\prime}} \varphi(\gamma)\right)$ for any signature morphism $\varphi: \Sigma \rightarrow \Sigma^{\prime}$.

The entailment systems of [30] or the $\pi$-institutions of [18] are $\mathcal{L}$-entailment systems when $\mathcal{L}$ is the two-valued Boolean algebra. Both of them represent multi-signature extensions, in the spirit of institution theory [23], of Tarski's abstract axiomatization of consequence relations [39] although the latter is formulated in terms of closure operators. Very related and important early work on abstract entailments is also due to Dana Scott [37]. The restriction of Dfn. 3.4 to a single signature (the main implication being the absence of Translation) is essentially the same as the 'graded consequences' of Chakraborty [4, 5]. Dfn. 3.4 comes very close to the so-called 'generalized entailment systems' of [16]; however here we do not assume a monad structure at the level of the abstract syntax. But the major difference with respect to the corresponding concept from [16] occurs in the rule Transitivity which in our Dfn. 3.4 
relies upon an abstract binary operation $*$ (which in the case of a residuated lattice is its monoidal operation) rather than meet operation $\wedge$, and essential aspect that our Dfn. 3.4 shares with the graded consequences of $[4,5]$. Of course, this is irrelevant when $*$ and $\wedge$ coincide, such as in the case of Heyting algebras, but it makes an important difference in the other situations. An important technical argument supporting the use of $*$ rather than $\wedge$ in Transitivity comes from the semantics; below we will see that in general the many-valued semantic consequence satisfies Transitivity only when formulated using *. It should be noted that the two-valued Translation appears in [18, 40] under the name structurality.

As noted in [15] the main stream many-valued/fuzzy logic tradition considers only crisp consequences, our work together with $[4,5,16,34]$ being exceptions. Graded entailments may be intuitively interpreted in various ways, as provability degree, as degree of confidence in proofs, or even as a(n inverse) measure for the complexity of a proof. The more complex a proof the lower its truth value in the lattice $\mathcal{L}$. In other words, the degree of confidence in a proof is decreasing monotonic with respect to the complexity of the respective proof. By assigning low truth values to certain generating proof rules we may be able to use the truth value of an entailment $E \vdash \Gamma$ to measure how much certain rules are used in a proof. There are also temporal interpretations of graded proofs (see Ex. 4.4 below).

\subsection{Graded proofs}

Fact 3.2. For any fixed logic syntax, any partial order $\mathcal{L}=(L, \leq)$ induces a canonical partial order on the class of the respective $\mathcal{L}$-rules systems defined by

$$
\vdash \leq \vdash^{\prime} \text { if and only if for each signature } \Sigma \text { and any } E \subseteq \operatorname{Sen}(\Sigma), \gamma \in \operatorname{Sen}(\Sigma),\left(E \vdash_{\Sigma} \gamma\right) \leq\left(E \vdash_{\Sigma}^{\prime} \gamma\right) \text {. }
$$

Moreover, if $\mathcal{L}$ is a meet-semilattice then any family $\left(r^{i}\right)_{i \in I}$ of $\mathcal{L}$-rules systems that share a common logic syntax has greatest lower bound $\vdash$ defined by

$$
E \vdash_{\Sigma} \gamma=\bigwedge_{i}\left(E r_{\Sigma}^{i} \gamma\right)
$$

Proposition 3.1. Let us asume that $\mathcal{L}$ is like in Def. 3.4 and in addition $*$ is increasing monotonic. Then the greatest lower bound of any family of $\mathcal{L}$-entailment systems that share a common logic syntax is an $\mathcal{L}$-entailment system.

Proof. Let (Sign, Sen, $\left.\left(\vdash_{\Sigma}^{i}\right)_{\Sigma \in|\operatorname{Sign}|}\right)_{i \in I}$ be a family of $\mathcal{L}$-entailment systems that share a common logic syntax. Let (Sign, Sen, $\left(t_{\Sigma}\right)_{\Sigma \in \mid \text { Sign } \mid}$ ) denote their greatest lower bound according to Fact 3.2. We have to show that this satisfies the axioms of Dfn. 3.4.

(Reflexivity) This is trivial.

(Monotonicity) Let $E \subseteq E^{\prime} \subseteq \operatorname{Sen}(\Sigma)$. Then

$$
\begin{aligned}
E \vdash \gamma & =\bigwedge_{i}\left(E \vdash^{i} \gamma\right) & & (\text { by Fact 3.2) } \\
& \leq \bigwedge_{i}\left(E^{\prime} \vdash^{i} \gamma\right) & & \left(\text { by Monotonicity for each } \vdash^{i}\right) \\
& =E^{\prime} \vdash \gamma & & \text { (by Fact 3.2). }
\end{aligned}
$$

(Transitivity) Let $E, \Gamma \subseteq \operatorname{Sen}(\Sigma)$ and $\gamma^{\prime} \in \operatorname{Sen}(\Sigma)$. Then

$$
\begin{aligned}
(E \vdash \Gamma) *\left(\Gamma \vdash \gamma^{\prime}\right) & =\bigwedge_{i}\left(E \vdash^{i} \Gamma\right) * \bigwedge_{i}\left(\Gamma \vdash^{i} \gamma^{\prime}\right) & & \text { (by Fact 3.2) } \\
& \leq \bigwedge_{i}\left(\left(E \vdash^{i} \Gamma\right) *\left(\Gamma \vdash^{i} \gamma^{\prime}\right)\right) & & \text { (by the monotonicity of } *) \\
& \leq \bigwedge_{i}\left(E \vdash^{i} \gamma^{\prime}\right) & & \text { (by Transitivity for each } \left.\vdash^{i}\right) \\
& =E \vdash \gamma^{\prime} & & \text { (by Fact 3.2). }
\end{aligned}
$$

(Translation) Let $E \subseteq \operatorname{Sen}(\Sigma), \gamma \in \operatorname{Sen}(\Sigma)$ and $\varphi: \Sigma \rightarrow \Sigma^{\prime} \in \operatorname{Sign}$. Then

$$
\begin{aligned}
E \vdash \gamma & =\bigwedge_{i}\left(E \vdash^{i} \gamma\right) & & (\text { by Fact 3.2) } \\
& \leq \bigwedge_{i}\left(\varphi(E) \vdash^{i} \varphi(\gamma)\right) & & \left(\text { by Translation for each } \vdash^{i}\right) \\
& =\varphi(E) \vdash \varphi(\gamma) & & \text { (by Fact 3.2). }
\end{aligned}
$$

An immediate consequence of Prop. 3.1 is the possibility to provide the following definition. 
Definition 3.5. For $\mathcal{L}$ like in Prop. 3.1, the $\mathcal{L}$-entailment system generated by an $\mathcal{L}$-rules system is the least $\mathcal{L}$ entailment system greater than the respective $\mathcal{L}$-rules system.

Example 3.3. In continuation of Ex. 3.1 let us consider a signature $\Sigma$ consisting of three operation symbols, namely a constant 0 , a unary function $s$, and a binary function _ $+_{\text {. }}$. Let $E$ consists of the following two axioms:

$$
\begin{array}{ll}
a 1: & (\forall x) x+0>x . \\
a 2: & (\forall x, y) x+(s y)>s(x+y) .
\end{array}
$$

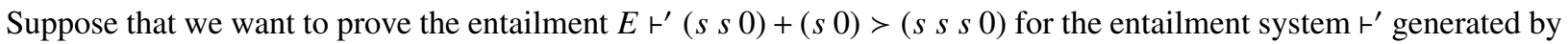
the above rule system. The proof goes as follows:

$$
\begin{aligned}
& (I 1) \quad a 2 \vdash^{\prime}(s s 0)+(s 0)>s((s s 0)+0) \\
& \text { (I2) } \quad a 1 \quad{ }^{\prime}(s s 0)+0>(s s 0) \\
& \text { (I3) } \quad E \quad r^{\prime}(s s 0)+(s 0)>s((s s 0)+0) \\
& \text { (I4) } \quad E \quad{ }^{\prime}(s s 0)+0>(s s 0) \\
& \text { (I5) } \quad(s s 0)+0>(s s 0) \quad r^{\prime} s((s s 0)+0)>(s s s 0) \\
& \text { (I6) } \quad r^{\prime} s((s s 0)+0)>(s s s 0) \\
& \text { (I7) } E \quad \vdash^{\prime} \Gamma=\left\{\left(\begin{array}{l}
s \\
s
\end{array}\right)+(s 0)>s((s, 0)+0)\right. \text {, } \\
& \left.\left.s((s s 0)+0)>\left(\begin{array}{ll}
s s & s
\end{array}\right)\right\} \quad \geq \frac{1}{n} \quad \text { (from }(I 3),(I 6)\right) \\
& \text { (I8) } \quad \Gamma \quad r^{\prime}(s s 0)+(s 0)>(s \text { s } s \quad 0) \\
& E \quad \vdash^{\prime}(s s 0)+(s 0)>(s \text { s } s 0) \\
& \begin{array}{ll}
\geq \frac{1}{n} & (\text { by }(T))
\end{array} \\
& \geq \frac{1}{n} * 1=\frac{1}{n} \\
& \text { (from (I7), (I8) by Transitivity). }
\end{aligned}
$$

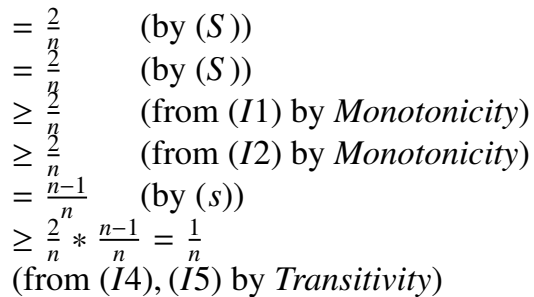

Example 3.4. In continuation of Ex. 3.2, let $\vdash^{\prime}$ be the $\mathcal{L}$-entailment system generated by $M P$ and $B$. By Transitivity we get that

$$
\{q, q \Rightarrow p\} \vdash^{\prime} \neg \square p \geq 0.9 * 0.8=0.7
$$

\section{4. $\mathcal{L}$-institutions}

In this section

1. We introduce the main concept of many-valued institution as a fully abstract many-valued model theory and show how this gives rise naturally to a Galois connection between the syntax and the semantics.

2. We present several logical systems captured as many-valued institutions. All of them are many-valued logics except one, namely classical temporal logic that arises naturally as a many-valued institution.

3. We define a graded notion of semantic consequence at the level of abstract many-valued institutions which we show that satisfies the axioms of graded entailment systems discussed in the previous section. Moreover, we show that this interpretation of many-valued institutions as graded entailment systems is a retract.

4. We define what means that a graded rules or entailment system is sound with respect to a many-valued institution and prove that the soundness property of a system of rules transfers automatically to its generated entailment system.

\subsection{The main concept}

Definition 4.1 ( $\mathcal{L}$-institution). Given a set $L$, called the space of the truth values, an $L$-institution $\mathcal{I}=\left(\operatorname{Sign}^{\mathcal{I}}, \operatorname{Sen}^{\mathcal{I}}, \operatorname{Mod}^{\mathcal{I}},\left(\models_{\Sigma}^{\mathcal{I}}\right.\right.$ )$_{\left.\Sigma \in \mid \operatorname{Sign}^{I_{1}}\right)}$ consists of

- a category $\operatorname{Sign}^{I}$ whose objects are called signatures,

- a functor $\operatorname{Sen}^{I}: \operatorname{Sign}^{I} \rightarrow$ Set giving for each signature a set whose elements are called sentences over that signature, 
- a functor $\operatorname{Mod}^{I}:\left(\operatorname{Sign}^{I}\right)^{o p} \rightarrow \mathbf{C A T}$, giving for each signature $\Sigma$ a category whose objects are called $\Sigma$-models, and whose arrows are called $\Sigma$-(model) homomorphisms, and

- a L-valued relation $\models_{\Sigma}^{I}:\left|\operatorname{Mod}^{I}(\Sigma)\right| \times \operatorname{Sen}^{I}(\Sigma) \rightarrow$ Lfor each $\Sigma \in\left|\operatorname{Sign}^{I}\right|$, called the satisfaction relation, such that for each morphism $\varphi: \Sigma \rightarrow \Sigma^{\prime} \in \operatorname{Sign}^{I}$, the Satisfaction Condition

$$
\left(M^{\prime} \vDash_{\Sigma^{\prime}}^{I} \operatorname{Sen}^{I}(\varphi)(\rho)\right)=\left(\operatorname{Mod}^{I}(\varphi)\left(M^{\prime}\right) \vDash_{\Sigma}^{I} \rho\right)
$$

holds for each $M^{\prime} \in\left|\operatorname{Mod}^{I}\left(\Sigma^{\prime}\right)\right|$ and $\rho \in \operatorname{Sen}^{I}(\Sigma)$.

For $\mathcal{L}=(L, \leq)$ partial order, an $\mathcal{L}$-institution means just an L-institution.

The Satisfaction Condition says that the truth degree is an invariant with respect to change of notation. Note that when presenting the satisfaction relation $\vDash=$ as a natural transformation $\operatorname{Sen} \Rightarrow[|\operatorname{Mod}(-)| \rightarrow L]$, where $[|\operatorname{Mod}(\Sigma)| \rightarrow L]$ denotes the set of the functions from $|\operatorname{Mod}(\Sigma)|$ to $L$ and for any signature morphism $\varphi: \Sigma \rightarrow \Sigma^{\prime},[|\operatorname{Mod}(\varphi)| \rightarrow L](f)=$ $f \circ|\operatorname{Mod}(\varphi)|$, the Satisfaction Condition (12) is just the naturality property of $\models$ :

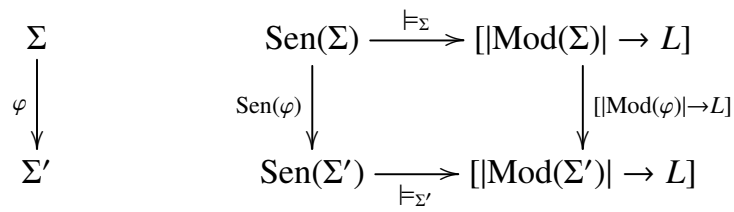

Classic institutions [23] are covered by Dfn. 4.1 when $L$ consists of two elements. The step from classic two-valued institutions to many-valued institutions is hardly new; in a form similar to Dfn. 4.1 this idea had appeared already in the so-called 'galleries' of [29]. The recently introduced 'generalized institutions' of [16] are very similar to Dfn. 4.1, however they introduce an additional monadic structure on the sentence functor meant to model substitution systems. A fully abstract treatment of many-valued semantics appears very early in [34], however it differs form the approach of $\mathcal{L}$-institutions in two quite important aspects. One is the single-signature feature of [34]. The other is that in [34] there is a collapse of model theory modulo elementary equivalence, which makes it unusable for the development of a proper fully abstract many-valued model theory. In other words, Pavelka's approach in [34] would correspond to an $\mathcal{L}$-institution that has only one signature $\Sigma$ and also such that $|\operatorname{Mod}(\Sigma)| \subseteq L^{\operatorname{Sen}(\Sigma)}$ (with $M \vDash \rho$ being just $M(\rho)$ ).

It would be possible to make Dfn. 4.1 more general by letting the space $L$ of truth values float by making $L$ a component of the concept of signature (somehow in the spirit of [29]). However because of reasons of simplicity of presentation we refrain here from that kind of generalisation, which anyway may be canonically achieved from Dfn. 4.1 by a Grothendieck flattening in the style of [7].

The following Galois connection generalizes a similar situation from [23] to many-valued truth.

Definition 4.2. Let $\mathcal{L}$ be a complete meet-semilattice. In any $\mathcal{L}$-institution:

- For any $\Sigma$-model $M$ we let the theory $M^{*}: \operatorname{Sen}(\Sigma) \rightarrow L$ such that $M^{*}(\rho)=(M \vDash \rho)$. For any class of models $\mathcal{M} \subseteq|\operatorname{Mod}(\Sigma)|$ we let $\mathcal{M}^{*}=\bigwedge_{M \in \mathcal{M}} M^{*}$.

- For any $\Sigma$-theory $X: \operatorname{Sen}(\Sigma) \rightarrow L$ we let $X^{*}=\left\{M \in|\operatorname{Mod}(\Sigma)| \mid X \leq M^{*}\right\}$.

Fact 4.1. For each signature $\Sigma$, the mappings (_)* of Dfn. 4.2 define a Galois connection between $(\mathcal{P}(|\operatorname{Mod}(\Sigma)|), \supseteq)$ and $\left(L^{\operatorname{Sen}(\Sigma)}, \leq\right)$.

Definition 4.3 (Consistency). In any $\mathcal{L}$-institution, a $\Sigma$-theory $X$ is consistent when $X^{*} \neq \emptyset$.

\subsection{Examples of $\mathcal{L}$-institutions}

The examples below are given rather briefly, for full details the reader may go to the recommended references. In all examples we assume a fixed residuated lattice $\mathcal{L}$. However the examples can be truncated such that they accomodate poorer order theoretic structures for $\mathcal{L}$. 
Example 4.1 (Propositional many-valued logic). The signatures of this $\mathcal{L}$-institution are just sets (of propositional variables). Signature morphism are functions. For any set $P$, the $P$-sentences are terms over $P$ built by the usual logical connectives $\mathrm{\top}$ (true),$\perp$ (false), $\wedge$ (conjunction), $\vee$ (disjunction), $\Rightarrow$ (implication) plus $*$. A $P$-model $M$ is just a valuation $M: P \rightarrow L$. For any function $\varphi: P \rightarrow P^{\prime}$, the reduct of a $P^{\prime}$-model $M^{\prime}$ is just $M \circ \varphi$. The satisfaction relation $M \vDash \rho$ is computed by induction on the structure of $\rho$ from the valuation $M$ by using the operations of $\mathcal{L}$.

Example 4.2 (First order many-valued logic). The full details of this $\mathcal{L}$-institution in its many sorted form may be found in [10, 12, 14]. For simplicity, here we briefly present it in its single sorted form, which also accords with [25].

A signature is a pair $(F, P)$ where $F=\left(F_{n}\right)_{n \in \omega}$ and $P=\left(P_{n}\right)_{n \in \omega}$ are families of sets of operation and relation symbols indexed by arity. Signature morphisms $\varphi:(F, P) \rightarrow\left(F^{\prime}, P^{\prime}\right)$ consists of two components, one for the operations and the other for the relations, each of them being a family of functions indexed by the arities.

$(F, P)$-sentences are constructed from atoms of the form $\pi\left(t_{1}, \ldots, t_{n}\right)$ where $\pi \in P_{n}$ and $t_{1}, \ldots, t_{n}$ are $F$-terms, by the propositional logical connectives $\top, \perp, \wedge, \vee, \Rightarrow, *$ plus quantifiers $(\forall X)$ and $(\exists X)$ for each finite set $X$ of variables. In the case of the quantifiers, if $\rho$ is an $(F+X, P)$-sentence (where $F+X$ adds $X$ to $F$ as new constants, i.e. symbols of arity 0$)$ then $(\forall X) \rho$ and $(\exists X) \rho$ are $(F, P)$-sentences.

An $(F, P)$-model $M$ consists of a carrier set $|M|$ and an interpretation of the operation symbols $\sigma \in F_{n}$ as functions $M_{\sigma}:|M|^{n} \rightarrow|M|$ and of the relation symbols $\pi \in P_{n}$ as $L$-relations on $|M|$, i.e. $M_{\pi}:|M|^{n} \rightarrow L$. For any signature morphism $\varphi:(F, P) \rightarrow\left(F^{\prime}, P^{\prime}\right)$ the reduct of an $\left(F^{\prime}, P^{\prime}\right)$-model $M^{\prime}$ is the $(F, P)$-model $M$ defined by $|M|=\left|M^{\prime}\right|$ and $M_{x}=M_{\varphi(x)}^{\prime}$ for each operation or relation symbol $x$ in $(F, P)$.

The satisfaction relation $M \vDash \rho$ is computed by induction on the structure of $\rho$ as follows. For each atom $\pi\left(t_{1}, \ldots, t_{n}\right),\left(M=\pi\left(t_{1}, \ldots, t_{n}\right)\right)=M_{\pi}\left(M_{t_{1}}, \ldots, M_{t_{n}}\right)$, where for each $F$-term $\sigma\left(t_{1}, \ldots, t_{k}\right), M_{\sigma\left(t_{1}, \ldots, t_{k}\right)}=M_{\sigma}\left(M_{t_{1}}, \ldots, M_{t_{k}}\right)$. For the logical connectives we compute the respective value in $\mathcal{L}$, i.e. $\left(M \vDash \rho_{1} \wedge \rho_{2}\right)=\left(M \vDash \rho_{1}\right) \wedge\left(M \vDash \rho_{2}\right)$ and similarly for $\vee, \Rightarrow, *$. For the quantifiers $(M \vDash(\forall X) \rho)=\bigwedge\left\{M^{\prime} \vDash \rho \mid\right.$ the reduct of $M^{\prime}$ to $(F, P)$ is $\left.M\right\}$, and similarly for the existential quantification but considering the supremum rather than infimum.

Example 4.3 (Horn many-valued logic). This $\mathcal{L}$-institution, which appears implicitly in [11], is obtained from manyvalued first order logic just by restricting the sentences to the Horn ones, i.e. sentences of the form $(\forall X) H \Rightarrow C$ where $C$ is an atom and $H$ is a quantifier-free sentence formed from atoms and the connectives $\wedge, \vee, *$. In [11] it is shown that theories of this institution admit initial semantics.

Example 4.4 (Propositional temporal logic). We fix a complete total order $\mathcal{L}=(L, \leq)$, that models the 'time'. The signatures of this $\mathcal{L}$-institution are the sets, just like in the Ex. 4.1 of propositional many-valued logic. For any set $P$, the $P$-sentences are like in Ex. 3.2 and Ex. 3.4. A $P$-model $M$ consists of an interpretation $M_{\pi} \subseteq L$ for each $\pi \in P$. For any function $\varphi: P \rightarrow P^{\prime}$, the $\varphi$-reduct $\operatorname{Mod}(\varphi)\left(M^{\prime}\right)$ of a $P^{\prime}$-model $M^{\prime}$ is defined by $\left(\operatorname{Mod}(\varphi)\left(M^{\prime}\right)\right)_{\pi}=M_{\varphi(\pi)}^{\prime}$. For each $P$-model $M$, each $w \in L$, and each $P$-sentences $\rho$ we define $\left(M F^{w} \rho\right) \in\{0,1\}$ by induction on the structure of $\rho$ as follows:

- for each $\pi \in P,\left(M \models^{w} \pi\right)=1$ if and only if $w \in M_{\pi}$;

$-\left(M \models^{w} \rho_{1} \wedge \rho_{2}\right)=1$ if and only if $\left(M \models^{w} \rho_{1}\right)=1$ and $\left(M \models^{w} \rho_{2}\right)=1$ and similarly for $\vee, \Rightarrow, \neg$;

- $\left(M \models^{w} \square \rho\right)=1$ if and only if for any $w \leq w^{\prime} \in|M|$ we have that $\left(M \vDash^{w^{\prime}} \rho\right)=1$.

For any $P$-model $M$ and any $P$-sentence $\rho$ we then define

$$
\left(M \models_{P} \rho\right)=\bigvee\left\{w \in L \mid \forall w^{\prime} \leq w, M \vDash^{w^{\prime}} \rho\right\} .
$$

The Satisfaction Condition (12) follows swiftly from the relation $\left(M^{\prime} \vDash^{w} \operatorname{Sen}(\varphi)(\rho)\right)=\left(\operatorname{Mod}(\varphi)\left(M^{\prime}\right) \vDash^{w} \rho\right)$, which gets a straightforward proof by induction on the structure of $\rho$.

Another way to define $\left(M \models_{P} \rho\right)$, that fits the way satisfaction is commonly considered in linear temporal logic $(L T L)$ [17] is given by the formula

$$
\left(M \models_{P} \rho\right)=\bigwedge\left\{w \in L \mid M \vDash^{w} \rho\right\}
$$

This represents a kind of inverse degree of satisfaction, that gives the first moment when $\rho$ holds. Then $M \models_{P}^{L T L} \rho$ if and only if $\left(M \models_{P} \rho\right)=\perp$. 
Example 4.5 (Fuzzy multi-algebras). Multi-algebras have been introduced as an algebraic specification framework for non-determinism [26, 41, 42] and later on developed as a general framework for algebraic specification [27]. Lamo's thesis [27] shows that multi-algebras subsume important algebraic formalisms used in formal specification such as partial algebra [3] and membership algebra [31]. The non-deterministic nature of the operations in multialgebras makes the logic of multi-algebras very suitable for a fuzzy generalization; this has been realized in [14] as the so-called $\mathcal{L}$-institution the fuzzy multi-algebras. Here we briefly recall it in its single rather than many sorted form.

The signatures are pairs $(F, C)$ where $F=\left(F_{n}\right)_{n \in \omega}$ is a family of sets of operation symbols and $C$ is a set of deterministic constants. Signature morphisms maps the two components in a compatible way like in the previous examples.

$(F, C)$-sentences are formed from atoms $t<t^{\prime}$ (with $t$ and $t^{\prime}$ being $(F+C$ )-terms) by iterative applications of connectives $(\wedge, \vee, \Rightarrow, *)$ and quantifications just like in first order many-valued logic.

An $(F, C)$-model $M$ consists of a carrier set $|M|$, for each $\sigma \in F_{n}$ a function $M_{\sigma}:|M|^{n+1} \rightarrow L$, and for each deterministic constant $c \in C_{s}$, an element $M_{c} \in|M|$. Intuitively, $M_{\sigma}\left(x_{1}, \ldots, x_{n}, x_{n+1}\right)$ is thought as the truth degree of $\sigma\left(x_{1}, \ldots, x_{n}\right)=x_{n+1}$ in $M$. Model reducts are similar to those in the previous examples.

For defining the satisfaction between models and sentences we first define for each $(F, C)$-model $M$ a term evaluation function

$$
M\left[\sigma\left(t_{1}, \ldots, t_{n}\right), a\right]=\left\{\begin{array}{l}
\top, \text { when } \sigma \in C, M_{\sigma}=a \\
\perp, \text { when } \sigma \in C, M_{\sigma} \neq a \\
\vee\left\{\left.M_{\sigma}\left(b_{1}, \ldots, b_{n}, a\right) \wedge M\left[t_{1}, b_{1}\right] \wedge \ldots \wedge M\left[t_{n}, b_{n}\right]\left|\left(b_{1}, \ldots, b_{n}\right) \in\right| M\right|^{n}\right\}, \text { when } \sigma \in F_{n} .
\end{array}\right.
$$

Then we define $M \vDash \rho$ by induction on the structure of $\rho$ just like in many-valued first order logic, but starting from $\left(M \vDash t<t^{\prime}\right)=\bigwedge\left\{M[t, a] \Rightarrow M\left[t^{\prime}, a\right] \mid a \in M\right\}$.

Example 4.6 (Abstract many-valued logic of [14]). This generic $\mathcal{L}$-institution, denoted $\mathcal{I}(\mathcal{L})$, has been developed in [14] as a canonical model theoretic framework over abstract logic syntaxes. In [14] it is shown that propositional many-valued, first order many-valued, and fuzzy multi-algebras can be conservatively embedded into $\mathcal{I}(\mathcal{L})$, which means that their semantics may be replaced by the generic categorical one provided by $\mathcal{I}(\mathcal{L})$. Moreover in [14] it is proved that $\mathcal{I}(\mathcal{L})$ enjoys naturally model amalgamation and the method of diagrams, which represent two of the most fundamental institutional model theoretic properties [10].

\subsection{Semantic consequence in $\mathcal{L}$-institutions}

Definition 4.4. Given an $\mathcal{L}$ institution such that $\mathcal{L}$ is a complete meet-semilattice, for each $\Sigma$-model $M$ and each set E of $\Sigma$-sentences we define

$$
\left(M \vDash_{\Sigma} E\right)=\bigwedge\left\{M \models_{\Sigma} \rho \mid \rho \in E\right\} .
$$

Corollary 4.1. In any $\mathcal{L}$ institution such that $\mathcal{L}$ is a complete meet-semilattice, for any signature morphism $\varphi: \Sigma \rightarrow$ $\Sigma^{\prime}$, for any set $E$ of $\Sigma$-sentences and any $\Sigma^{\prime}$-model $M^{\prime}$ we have that

$$
\left(\operatorname{Mod}(\varphi)\left(M^{\prime}\right) \vDash_{\Sigma} E\right)=\left(M^{\prime} \vDash_{\Sigma^{\prime}} \operatorname{Sen}(\varphi)(E)\right)
$$

Definition 4.5 (Semantic consequence). Given an $\mathcal{L}$-institution such that $\mathcal{L}$ is a complete residuated lattice, for any $E \subseteq \operatorname{Sen}(\Sigma)$ and any $\gamma \in \operatorname{Sen}(\Sigma)$ we define

$$
\left(E \vDash_{\Sigma} \gamma\right)=\bigwedge\left\{\left(M \vDash_{\Sigma} E\right) \Rightarrow\left(M \vDash_{\Sigma} \gamma\right)|M \in| \operatorname{Mod}(\Sigma) \mid\right\} .
$$

This concept of semantic consequence, which subsumes the semantic consequence in classical two-valued institutions [10,23], appears in a disguised form in [34] within the context of Pavelka's theory of fuzzy consequence operators and in a form that is more explicitly similar to ours in [5] within the framework of 'graded consequence relations'. However the semantic frameworks of [34] and [5] are very similar and as we have argued above less general than ours, in both of them models being in fact fuzzy theories. In [15] it is argued that this is the only non-crisp semantic consequence concept in the many-valued/fuzzy logic literature.

By (4) of Prop. 2.1 we have the following: 
Fact 4.2. For any sets $E, \Gamma$ of $\Sigma$-sentences

$$
\left(E \vDash_{\Sigma} \Gamma\right)=\bigwedge\left\{\left(M \vDash_{\Sigma} E\right) \Rightarrow\left(M \vDash_{\Sigma} \Gamma\right)|M \in| \operatorname{Mod}(\Sigma) \mid\right\} .
$$

Proposition 4.1 (Semantic entailment). The semantic consequence of an $\mathcal{L}$-institution is an $\mathcal{L}$-entailment system, called the semantic entailment system of $\mathcal{I}$.

Proof. We check one by one the axioms given by Def. 3.4.

(Reflexivity) For each model $M$ by (1) we have that $(M \vDash \gamma) \Rightarrow(M \vDash \gamma)=\mathrm{T}$. Hence $(\{\gamma\} \vDash \gamma)=\mathrm{T}$.

(Monotonicity) Let $E \subseteq E^{\prime} \subseteq \operatorname{Sen}(\Sigma)$. For each $\Sigma$-model $M$, because $E \subseteq E^{\prime}$ from Dfn. 4.4 it follows that

$$
\left(M \vDash E^{\prime}\right) \leq(M \vDash E) .
$$

From (15) and (2) (of Prop. 2.1) it follows that

$$
\left(M \vDash E^{\prime}\right) \Rightarrow(M \vDash \gamma) \leq(M \vDash E) \Rightarrow(M \vDash \gamma)
$$

hence by (3) (of Prop. 2.1) it follows that $\left(E^{\prime} \vDash \gamma\right) \leq(E \vDash \gamma)$.

(Transitivity) Let $E, \Gamma \subseteq \operatorname{Sen}(\Sigma)$ and $\gamma^{\prime} \in \operatorname{Sen}(\Sigma)$.

$$
\begin{aligned}
(E \models \Gamma) *\left(\Gamma \models \gamma^{\prime}\right) & =\bigwedge_{M}((M \models E) \Rightarrow(M \vDash \Gamma)) * \bigwedge_{M}\left((M \models \Gamma) \Rightarrow\left(M \models \gamma^{\prime}\right)\right) & \text { (by Dfn. } 4.5 \text { and Fact 4.2) } \\
& \leq \bigwedge_{M}\left(((M \models E) \Rightarrow(M \models \Gamma)) *\left((M \models \Gamma) \Rightarrow\left(M \models \gamma^{\prime}\right)\right)\right) & \text { (by monotonicity of } *) \\
& \leq \bigwedge_{M}\left((M \models E) \Rightarrow\left(M \models \gamma^{\prime}\right)\right) & \text { (by (5) of Prop. 2.1) } \\
& =E \vDash \gamma^{\prime} & \text { (by Dfn. 4.5). }
\end{aligned}
$$

(Translation) Let $E \subseteq \operatorname{Sen}(\Sigma), \gamma \in \operatorname{Sen}(\Sigma)$ and let $\varphi: \Sigma \rightarrow \Sigma^{\prime} \in \operatorname{Sign}$.

$$
\begin{aligned}
E \models_{\Sigma} \gamma & =\bigwedge_{M}\left(\left(M \models_{\Sigma} E\right) \Rightarrow\left(M \models_{\Sigma} \gamma\right)\right) & & \text { (by Dfn. 4.5) } \\
& \leq \bigwedge_{M^{\prime}}\left(\left(M^{\prime} \vDash_{\Sigma^{\prime}} \varphi(E)\right) \Rightarrow\left(M^{\prime} \vDash_{\Sigma^{\prime}} \varphi(\gamma)\right)\right) & & \text { (by Cor. 4.1) } \\
& =\varphi(E) \vDash_{\Sigma^{\prime}} \varphi(\gamma) & & \text { (by Dfn. 4.5). }
\end{aligned}
$$

A similar result has been proved in [5] but within a single signature context. Note that the result of Prop. 4.1 subsumes the corresponding result from classical two-valued institution theory [10] that says that the semantic consequence of any institution is an entailment system.

The following result showing that the mapping from $\mathcal{L}$-institutions to $\mathcal{L}$-entailment systems given by $\mathrm{Dfn}$. 4.5 and Prop. 4.1 is a retract will be used later in the paper to invoke semantic theoretic arguments within entailment theoretic frameworks.

Proposition 4.2. Let $\mathcal{L}$ be complete residuated lattice. For any $\mathcal{L}$-entailment system (Sign, Sen, $\left.\left(\vdash_{\Sigma}\right)_{\Sigma \in|\operatorname{Sign}|}\right)$ there exists an $\mathcal{L}$-institution (Sign, Sen, Mod, $\left(\models_{\Sigma}^{\bullet}\right)_{\Sigma \in|\operatorname{Sign}|}$ ) such that its semantic entailment system $\models^{\bullet}$ coincides with $\vdash$.

Proof. We define the model functor Mod as follows:

- $\operatorname{Mod}(\Sigma)$ consists of pairs $M=\left(\psi_{M}, E_{M}\right)$ consisting of a signature morphism $\psi_{M}: \Sigma \rightarrow \Omega_{M}$ and a set $E_{M}$ of $\Omega_{M}$-sentences;

- for each signature morphism $\varphi: \Sigma \rightarrow \Sigma^{\prime}$ the reduct $\operatorname{Mod}(\varphi)\left(\psi_{M}, E_{M}\right)$ is defined as $\left(\varphi ; \psi_{M}, E_{M}\right)$.

The satisfaction relation is defined as

$$
\left(\left(\psi_{M}, E_{M}\right) \vDash^{\bullet} \rho\right)=\left(E_{M} \vdash \psi_{M}(\rho)\right) .
$$

The satisfaction condition follows immediately from the functoriality of Sen. Now we show that $(E \vdash \rho)=\left(E \vDash \vDash^{\bullet} \rho\right)$. We have that

$$
\left(E \models^{\bullet} \rho\right)=\bigwedge_{M}\left(\left(M \models^{\bullet} E\right) \Rightarrow\left(M \vDash^{\bullet} \rho\right)\right)=\bigwedge_{M}\left(\left(E_{M} \vdash \psi_{M}(E)\right) \Rightarrow\left(E_{M} \vdash \psi_{M}(\rho)\right)\right) .
$$


On the one hand, for each $M$

$$
\begin{aligned}
\left(E_{M} \vdash \psi_{M}(E)\right) *(E \vdash \rho) & \leq\left(E_{M} \vdash \psi_{M}(E)\right) *\left(\psi_{M}(E) \vdash \psi_{M}(\rho)\right) & & \text { (by Translation of } \vdash \text { and monotonicity of } *) \\
& \leq E_{M} \vdash \psi_{M}(\rho) & & \text { (by Transitivity of } \vdash) .
\end{aligned}
$$

Hence $(E \vdash \rho) \leq\left(E_{M} \vdash \psi_{M}(E)\right) \Rightarrow\left(E_{M} \vdash \psi_{M}(\rho)\right)$. This implies $(E \vdash \rho) \leq(E \vDash \bullet \rho)$.

On the other hand,

$$
\begin{aligned}
\left(E \vdash^{\bullet} \rho\right) & =\bigwedge_{M}\left(\left(E_{M} \vdash \psi_{M}(E)\right) \Rightarrow\left(E_{M} \vdash \psi_{M}(\rho)\right)\right. & & \\
& \leq((E \vdash E) \Rightarrow(E \vdash \rho)) & & \text { (by considering } \left.M=\left(1_{\Sigma}, E\right)\right) \\
& =(\top \Rightarrow(E \vdash \rho)) & & \text { (by Reflexivity and Monotonicity of } \vdash) \\
& =(E \vdash \rho) & & \text { (by (8) of Prop. 2.1). }
\end{aligned}
$$

\subsection{Soundness}

Definition 4.6 (Soundness). Let $\mathcal{L}$ be a complete residuated lattice. An $\mathcal{L}$-rules system (Sign, Sen, $\left.\left(\vdash_{\Sigma}\right)_{\Sigma \in|\operatorname{Sign}|}\right)$ is sound for an $\mathcal{L}$-institution (Sign, Sen, $\left.\operatorname{Mod},\left(\left.\right|_{\Sigma}\right)_{\Sigma \in|\operatorname{Sign}|}\right)$ when for each signature $\Sigma$ and any $E \subseteq \operatorname{Sen}(\Sigma), \gamma \in \operatorname{Sen}(\Sigma)$,

$$
\left(E \vdash_{\Sigma} \gamma\right) \leq\left(E \vDash_{\Sigma} \gamma\right) \text {. }
$$

Dfn. 4.6 represents a natural generalization of the corresponding soundness concept from classical two-valued institution theory $[9,10]$. In a similar form to ours it has been formulated in [16] within the context of the so-called 'generalized entailment systems'.

Corollary 4.2. The $\mathcal{L}$-entailment system generated by a sound $\mathcal{L}$-rules system is sound too.

Cor. 4.2, which generalizes to many-valued truth a corresponding result from classical two-valued institution theory $[9,10]$, justifies the rather tacit practice of establishing the soundness of an entailment system by a finite process in which one checks only the soundness of a rules system that presents in a finite way the respective entailment system.

Example 4.7. Let us consider the logic syntax of Ex. 3.1. For each signature $\Sigma$, we let $\Sigma$-models $M$ be the first order many-valued logic models for the signature that adds a binary relation symbol $>$ to $\Sigma$ (we denote here the interpretation of $>$ by $>_{M}$ rather than $M_{\succ}$ like in Ex. 4.2) and such the following conditions hold:

$-x>_{M} x=\mathrm{T}$

$-\left(x>_{M} y\right) \wedge\left(y>_{M} z\right) \leq\left(x>_{M} z\right)$

- for each $\sigma \in \Sigma_{k}$,

$$
\left(\left(x_{1}>_{M} y_{1}\right) \wedge \cdots \wedge\left(x_{k}>_{M} y_{k}\right)\right) * \frac{n-1}{n} \leq\left(M_{\sigma}\left(x_{1}, \ldots, x_{k}\right)>_{M} M_{\sigma}\left(y_{1}, \ldots, y_{k}\right)\right) .
$$

The satisfaction of a $\Sigma$-sentence $\rho$ by $M$ is defined like in first order many-valued logic (Ex. 4.2) with the following definition for the satisfaction of the atoms:

$$
\left(M \models t>t^{\prime}\right)=\left(M_{t}>_{M} M_{t^{\prime}}\right)
$$

These give an $\mathcal{L}$-institution.

By virtue of Cor. 4.2 the soundness of the $\mathcal{L}$-entailment system generated by the $\mathcal{L}$-rules system of Ex. 3.1 can be now established only by checking the soundness of the rules $R, T, S$ and $\sigma$. The soundness of $R, T, \sigma$ follow from the three conditions imposed above on the models; we skip this rather straightforward checks here. The soundness of $S$, which reads as

$$
\frac{2}{n} \leq\left(M \vDash(\forall X) t>t^{\prime}\right) \Rightarrow\left(M \vDash \theta[t]>\theta\left[t^{\prime}\right]\right)
$$

for each model $M$, follows if we showed that for each model $M$

$$
\bigwedge\left\{\frac{2}{n} *\left(M_{t}^{\prime}>_{M} M_{t^{\prime}}^{\prime}\right) \mid M \text { reduct of } M^{\prime}\right\} \leq\left(M_{\theta[t]}>_{M} M_{\theta\left[t^{\prime}\right]}\right) .
$$

The latter relation can be established by considering $M^{\prime}$ such that for each variable $x \in X, M_{x}^{\prime}=M_{\theta[x]}$ (in this case $M_{t}^{\prime}=M_{\theta[t]}$ and also the same for $\left.t^{\prime}\right)$. 
Example 4.8. Let $\mathcal{L}$ be the space of the truth values of Łukasiewicz's infinitary logic. For the logic syntax of Ex. 3.2 and 3.4 we consider the models $M$ as defined by Ex. 4.4 but additionally constrained by the condition that $M_{p} \cap\{0.7,0.9\}=\emptyset$. We consider the satisfaction as defined in Ex. 4.4. This yields a sub-institution of the $\mathcal{L}$-institution of Ex. 4.4 for which the $\mathcal{L}$-entailment system of Ex. 3.4 is sound.

This soundness property followed by virtue of Cor. 4.2 if we showed that $M P$ and $B$ are sound rules. In the case of $M P$ we may establish that

$$
(\{q, q \Rightarrow p\} \vDash p)=1
$$

but we skip this here. The soundness of $B$ is obtained as follows. Let $M$ be any model such that $M_{p} \cap\{0.7,0.9\}=\emptyset$. From $0.7 \notin M_{p}$ it follows that

$$
(M \vDash \neg \square p) \geq 0.7
$$

and from $0.9 \notin M_{p}$ that

$$
(M \vDash \rho) \leq 0.9 .
$$

Then

$$
\begin{aligned}
0.8 *(M \vDash p) & \leq 0.8 * 0.9 & & \text { (by }(17)) \\
& =0.7 & & \text { (by the definition of Łukasiewicz's arithmetic conjunction) } \\
& \leq(M \vDash \neg \square p) & & \text { (by }(16)) .
\end{aligned}
$$

It follows that $0.8 \leq(M \vDash p) \Rightarrow(M \vDash \neg \square p)$ hence $0.8 \leq p \vDash \neg \square p$.

\section{5. $\mathcal{L}$-closure systems}

The structure of this section is as follows:

1. We introduce the concept of many-valued closure system.

2. We define a general closure of fuzzy theories under graded entailment and show that this determines naturally a closure system. Moreover we give a characterization of this closure in terms of an algebraic formula.

3. We define a semantic closure for fuzzy theories in many-valued institutions and we show that this determines a closure system. Then we use this for defining a second closure at the general level of graded entailment systems and show that this is stronger than the closure previously defined. Moreover we show that in the absence of signature morphisms this interpretation of many-valued institutions to many-valued closure systems is a retract.

4. In the final part of this section we draw some conclusions based on the taxonomy of mutual interpretations between many-valued institutions, entailment systems, and closure systems.

\section{1. $\mathcal{L}$-closure systems}

In the classical two-valued framework the closure or consequence operators [39] constitute an alternative equivalent concept for that of entailment system. In [34] Pavleka had used Tarski's closure operators on $\mathcal{L}$-sets in order to provide a suitable concept of consequence operator for the many-valued framework. On the one hand, the following definition extends the concept introduced in [34] to the multi-signature framework by adding the $C$-Translation axiom. On the other hand, it represents an extension a generalization of the $\pi$-institutions of [18] to the many-valued framework.

Definition 5.1 ( $\mathcal{L}$-closure systems). Given a partial order $\mathcal{L}=(L, \leq)$, an $\mathcal{L}$-closure system is a tuple $\left(\operatorname{Sign}, \operatorname{Sen},\left(C_{\Sigma}\right)_{\Sigma \in|\operatorname{Sign}|}\right)$ where

- (Sign, Sen) is a logic syntax, and

$-C_{\Sigma}: L^{\operatorname{Sen}(\Sigma)} \rightarrow L^{\operatorname{Sen}(\Sigma)}$ satisfying the following axioms:

1. (C-Reflexivity) $X \leq C_{\Sigma} X$ for each $X$,

2. (C-Monotonicity) $C_{\Sigma} X \leq C_{\Sigma} Y$ when $X \leq Y$, 
3. (C-Transitivity) $C_{\Sigma}\left(C_{\Sigma} X\right)=C_{\Sigma} X$,

4. (C-Translation) $\mathcal{C}_{\Sigma}\left(\operatorname{Sen}(\varphi) ; X^{\prime}\right) \leq \operatorname{Sen}(\varphi) ; C_{\Sigma^{\prime}}\left(X^{\prime}\right)$ for any signature morphism $\varphi: \Sigma \rightarrow \Sigma^{\prime}$.

While in the classical two-valued situation the equivalence between the entailment-style [30] and the closure-style $[18,39]$ formulations of abstract consequence relations can be established very easily, this is not the case for the graded consequence. In what follows we give two different natural interpretations of $\mathcal{L}$-entailment as $\mathcal{L}$-closure.

\subsection{Weak closure}

The following definition owes inspiration to Goguen's many-valued interpretation of Modus Ponens [21].

Definition 5.2. Let $\mathcal{L}$ be a partial order with a binary operation $*$. A theory $X: \operatorname{Sen}(\Sigma) \rightarrow L$ is weakly closed when for each entailment $E r_{\Sigma} \rho$,

$$
X(E) *(E \vdash \rho) \leq X(\rho) .
$$

The following shows that the concept of weakly closure of Dfn. 5.2 subsumes the classical two-valued closure in entailment systems. We skip the rather straightforward proof.

Fact 5.1. If $\mathcal{L}$ is the two-valued Boolean algebra and $*$ is the Boolean conjunction, then a theory $X$ is weakly closed if and only if $\rho \in X$ whenever $X \vdash \rho$.

Proposition 5.1. Let $\mathcal{L}$ be a complete meet-semilattice with a monotonic binary operation $*$. The intersection of any family $\left(X_{i}\right)_{i \in I}$ of weakly closed theories is a weakly closed theory too.

Proof. We check the closure condition for $\bigwedge_{i} X_{i}$.

$$
\begin{array}{rlrl}
\left(\bigwedge_{i} X_{i}\right)(E) *(E \vdash \rho) & =\left(\bigwedge_{i} X_{i}(E)\right) *(E \vdash \rho) & \\
& \leq \bigwedge_{i}\left(X_{i}(E) *(E \vdash \rho)\right) & & \text { (monotonicity of } *) \\
& \leq \bigwedge_{i} X_{i}(\rho) & & \left(X_{i} \text { weakly closed }\right) \\
& =\left(\bigwedge_{i} X_{i}\right)(\rho) . & &
\end{array}
$$

Proposition 5.2. For any signature morphism $\varphi: \Sigma \rightarrow \Sigma^{\prime}$, if $X^{\prime}$ is a weakly closed $\Sigma^{\prime}$-theory then $\varphi$; $X^{\prime}$ is a weakly closed $\Sigma$-theory.

Proof. We check the closure condition for $\varphi ; X^{\prime}$.

$$
\begin{aligned}
\left(\varphi ; X^{\prime}\right)(E) *\left(E \vdash_{\Sigma} \rho\right) & =X^{\prime}(\varphi(E)) *\left(E \vdash_{\Sigma} \rho\right) & & \\
& \leq X^{\prime}(\varphi(E)) *\left(\varphi(E) \vdash_{\Sigma^{\prime}} \varphi(\rho)\right) & & \text { (by Translation and monotonicity of } *) \\
& \leq X^{\prime}(\varphi(\rho)) & & \text { (since } X \text { is weakly closed). }
\end{aligned}
$$

Prop. 5.1 allows the following definition.

Definition 5.3. For any theory $X$ let $X^{\circ}$ denote the least weakly closed theory greater than $X$.

Proposition 5.3. The operator (_) ${ }^{\circ}$ of $D f n .5 .3$ defines an $\mathcal{L}$-closure system.

Proof. The first three axioms of $\mathcal{L}$-closure systems follow immediately from the definition of $X^{\circ}$. C-Translation follows from Prop. 5.2 by noticing that $\operatorname{Sen}(\varphi) ; X^{\prime} \leq \operatorname{Sen}(\varphi) ;\left(X^{\circ}\right)$.

Proposition 5.4. Let $\mathcal{L}$ be a completely distributive lattice $\mathcal{L}$ together with an associative continuous binary operation *. In any $\mathcal{L}$-entailment system, for each theory $X$,

$$
X^{\circ}(\rho)=\bigvee_{E}(X(E) *(E \vdash \rho)) .
$$


Proof. Let $\bar{X}(\rho)=\bigvee_{E}(X(E) *(E \vdash \rho))$. By considering $E=\{\rho\}$ in the definition of $\bar{X}(\rho)$, by Reflexivity of $\vdash$, we get that $X \leq \bar{X}$. Since $X \leq X^{\circ}$, by the monotonicity of $*$ (which is a consequence of continuity), we have that $X(E) *(E \vdash \rho) \leq X^{\circ}(E) *(E \vdash \rho)$. Since $X^{\circ}$ is closed we have that $X^{\circ}(E) *(E \vdash \rho) \leq X^{\circ}(\rho)$. Thus for each $E$, $\rho$ we have that $X(E) *(E \vdash \rho) \leq X^{\circ}(\rho)$ hence $\bar{X} \leq X^{\circ}$. If we showed that $\bar{X}$ is closed, then since $X^{\circ}$ is the closure of $X$ it would also follow that $X^{\circ}=\bar{X}$, hence $\bar{X}=X^{\circ}$.

The check of the closure condition for $\bar{X}$ goes as follows:

$$
\begin{aligned}
\bar{X}(E) *(E \vdash \rho) & =\left(\bigwedge_{e \in E} \bar{X}(e)\right) *(E \vdash \rho) & & \\
& =\left(\bigwedge_{e \in E} \bigvee_{\Gamma}(X(\Gamma) *(\Gamma \vdash e))\right) *(E \vdash \rho) & & (\text { definition of } \bar{X}) \\
& =\left(\bigvee_{\Gamma} \bigwedge_{e \in E}(X(\Gamma) *(\Gamma \vdash e))\right) *(E \vdash \rho) & & (\mathcal{L} \text { is completely distributive) } \\
& =\left(\bigvee_{\Gamma}\left(X(\Gamma) * \bigwedge_{e \in E}(\Gamma \vdash e)\right) *(E \vdash \rho)\right. & & (* \text { is meet-continuous) } \\
& =\left(\bigvee_{\Gamma}(X(\Gamma) *(\Gamma \vdash E)) *(E \vdash \rho)\right. & & \\
& =\bigvee_{\Gamma}((X(\Gamma) *(\Gamma \vdash E)) *(E \vdash \rho)) & & (* \text { is join-continuous) } \\
& =\bigvee_{\Gamma}(X(\Gamma) *((\Gamma \vdash E)) *(E \vdash \rho)) & & (* \text { is associative) } \\
& \leq \bigvee_{\Gamma}(X(\Gamma) *(\Gamma \vdash \rho)) & & \text { (by Transitivity of } \vdash) \\
& \bar{X}(\rho) & & \text { (definition of } \bar{X}) .
\end{aligned}
$$

\subsection{Strong closure}

Now we define a second closure system on theories of $\mathcal{L}$-entailment systems by resorting to semantics.

Proposition 5.5. In any $\mathcal{L}$-institution, the Galois connection of Dfn. 4.2 determines a (two-valued) closure system (Sign, |Mod $\left.\mid,\left(_{-}\right)^{* *}\right)$ and an $\mathcal{L}$-closure system (Sign, Sen, (_) $\left.{ }^{* *}\right)$.

Proof. The first three properties of closure systems follow from the general properties of a Galois connection. Let us now prove $C$-Translation for both presumed closure systems.

$C$-Translation for (Sign, $\mid$ Mod $\left.\mid,\left(_{-}\right)^{* *}\right)$ is proved as follows: for each signature morphism $\varphi: \Sigma \rightarrow \Sigma^{\prime}$ and each class $\mathcal{M}$ of $\Sigma$-models,

$$
\begin{aligned}
\left(\operatorname{Mod}(\varphi)\left(\mathcal{M}^{\prime}\right)\right)^{* *} & \subseteq\left(\operatorname{Mod}(\varphi)\left(\mathcal{M}^{* *}\right)\right)^{* *} & & \text { (by } C \text {-Reflexivity of }\left(_{-}\right)^{* *} \text { on models) } \\
& =\left(\operatorname{Sen}(\varphi) ; \mathcal{M}^{\prime * * *}\right)^{*} & & \text { (by the satisfaction condition) } \\
& =\left(\operatorname{Sen}(\varphi) ; \mathcal{M}^{\prime *}\right)^{*} & & \text { (by general Galois connection properties) } \\
& =\operatorname{Mod}(\varphi)\left(\mathcal{M}^{\prime * *}\right) & & \text { (by the satisfaction condition) }
\end{aligned}
$$

$C$-Translation for (Sign, Sen, $\left(\left(_{-}^{* *}\right)\right.$ is proved as follows: for each signature morphism $\varphi: \Sigma \rightarrow \Sigma^{\prime}$ and each $\Sigma^{\prime}$-theory $X^{\prime}$,

$$
\begin{aligned}
\left(\operatorname{Sen}(\varphi) ; X^{\prime}\right)^{* *} & \leq\left(\operatorname{Sen}(\varphi) ; X^{\prime * *}\right)^{* *} & & \text { (by } C \text {-Reflexivity of }\left(_{-}\right)^{* *} \text { on sentences) } \\
& =\left(\operatorname{Mod}(\varphi)\left(X^{\prime *}\right)\right)^{* * *} & & \text { (by the satisfaction condition) } \\
& =\left(\operatorname{Mod}(\varphi)\left(X^{\prime *}\right)\right)^{*} & & \text { (by general Galois connection properties) } \\
& =\operatorname{Sen}(\varphi) ;\left(X^{\prime * *}\right) & & \text { (by the satisfaction condition). }
\end{aligned}
$$

Given a fuzzy theory $X, X^{* *}$ is essentially the same with the semantic consequence of $X$ in [34].

Definition 5.4. In any $\mathcal{L}$-institution a $\Sigma$-theory is strongly closed when $X=X^{* *}$.

Proposition 5.6. Let $\mathcal{L}$ be a complete residuated lattice. In any $\mathcal{L}$-institution, for any $\Sigma$-theory $X$ we have that $X^{\circ} \leq X^{* *}$, where $X^{\circ}$ denotes the weak closure of $X$ under semantic consequence (see Dfn. 5.2). 
Proof. Since $X \leq X^{* *}$, the conclusion followed if we proved that $X^{* *}$ is closed in the sense of Dfn. 5.2 for the semantic entailment $=$.

$$
\begin{aligned}
X^{* *}(E) *(E \models \rho) & =X^{* *}(E) *\left(\bigwedge_{M}((M \models E) \Rightarrow(M \models \rho))\right) & & \text { (by Dfn. 4.5) } \\
& \leq X^{* *}(E) *\left(\bigwedge_{X \leq M^{*}}((M \models E) \Rightarrow(M \models \rho))\right) & & \\
& =\left(\bigwedge_{X \leq M^{*}} M \models E\right) *\left(\bigwedge_{X \leq M^{*}}((M \models E) \Rightarrow(M \models \rho))\right) & & \text { (by definition of } \left.X^{* *}\right) \\
& \leq \bigwedge_{X \leq M^{*}}((M \models E) *((M \models E) \Rightarrow(M \models \rho))) & & \text { (by monotonicity of } *) \\
& \leq \bigwedge_{X \leq M^{*}}(M \models \rho) & & (\mathcal{L} \text { is residuated lattice) } \\
& =X^{* *}(\rho) & & \text { (by definition of } \left.X^{* *}\right) .
\end{aligned}
$$

The following is a consequence of Prop. 5.5 and Prop. 4.2.

Corollary 5.1. Let $\mathcal{L}$ be a complete residuated lattice and let (Sign, Sen, $\left.\left(\vdash_{\Sigma}\right)_{\Sigma \in|\operatorname{Sign}|}\right)$ be an $\mathcal{L}$-entailment system. Then

$$
X^{\bullet}(\rho)=\bigwedge\left\{E \vdash \psi(\rho) \mid \psi: \Sigma \rightarrow \Sigma^{\prime}, E \subseteq \operatorname{Sen}\left(\Sigma^{\prime}\right), X(\gamma) \leq E \vdash \psi(\gamma) \text { for each } \gamma \in \operatorname{Sen}\left(\Sigma^{\prime}\right)\right\}
$$

defines an $\mathcal{L}$-closure system.

Definition 5.5. For $\mathcal{L}$ complete residuated lattice, in any $\mathcal{L}$-entailment system a $\Sigma$-theory $X$ is strongly closed when $X=X^{\bullet}$.

Like the weak closure, the concept of strong closure also subsumes the two-valued concept of closure under entailment.

Proposition 5.7. If $\mathcal{L}$ is the two-valued Boolean algebra, then a theory $X$ is strongly closed if and only if $\rho \in X$ whenever $X \vdash \rho$.

Proof. Let us first assume that $X=X^{\bullet}$ and that $X \vdash \rho$ and prove that $\rho \in X$. Since $X=X^{\bullet}$ it is enough to show that $\rho \in X^{\bullet}$ which means that $E \vdash \psi(\rho)$ for each $E$ such that $E \vdash \psi(X)$. From $X \vdash \rho$, by Translation we have that $\psi(X) \vdash \psi(\rho)$. Hence, if $E \vdash \psi(X)$ then it follows that $E \vdash \psi(\rho)$.

Now we prove the opposite implication. Let us assume that $\rho \in X$ whenever $X \vdash \rho$. We have to show that $\rho \in X^{\bullet}$ implies $\rho \in X$. Let $\rho \in X^{\bullet}$. This means that $E \vdash \psi(\rho)$ for each $E$ such that $E \vdash \psi(X)$. Let us consider $\psi$ identity and $E=X$. Then $X \vdash \rho$, hence $\rho \in X$.

Note that at the level of abstract $\mathcal{L}$-entailment systems the definition of weak closure requires weaker conditions than that of strong closures, namely complete meet-semilattice with $*$ monotonic versus complete residuated lattice.

Corollary 5.2. Let $\mathcal{L}$ be a complete residuated lattice. In any $\mathcal{L}$-entailment system, for any $\Sigma$-theory $X$ we have that $X^{\circ} \leq X^{\bullet}$.

Proof. By Prop. 4.2 we have that $X^{\circ}$ is the weak closure of $X$ under $\models^{\bullet}$ and that $X^{\bullet}=X^{* *}$ is the semantic closure of $X$ under $F^{\bullet}$. The conclusion now follows by Prop. 5.6.

The following interpretation of $\mathcal{L}$-closure systems as $\mathcal{L}$-institutions gets a rather straightforward proof which we skip here.

Fact 5.2. For any $\mathcal{L}$-closure system $(\operatorname{Sign}, \operatorname{Sen}, C)$ the tuple $\left(\operatorname{Sign}, \operatorname{Sen}, \operatorname{Mod}, \models^{C}\right)$ where $\operatorname{Mod}(\Sigma)=L^{\operatorname{Sen}(\Sigma)}$ and $\left(X \models^{C}\right.$ $\rho)=(C X) \rho$ is an $\mathcal{L}$-institution if and only if for each signature morphism $\varphi: \Sigma \rightarrow \Sigma^{\prime}$ and each $\Sigma^{\prime}$-theory $X^{\prime}$

$$
C_{\Sigma}\left(\varphi ; X^{\prime}\right)=\varphi ;\left(C_{\Sigma^{\prime}} X^{\prime}\right)
$$

Moreover this is a left-inverse to the interpretation of discrete $\mathcal{L}$-institutions to discrete $\mathcal{L}$-closure systems, given by Prop. 5.5, that maps theories $X$ to their semantic closures $X^{* *}$.

The condition (19) is a rather heavy one, not to be expected in general from $\mathcal{L}$-closure systems. One way to get around it is to restrict the discussion to discrete $\mathcal{L}$-closure systems, i.e. without signature morphisms, which are technically essentially the same as single single signature frameworks.

The following is an immediate consequence of Prop. 4.1 (giving the semantic entailment system) and Fact 5.2.

Corollary 5.3. Let $\mathcal{L}$ be a complete residuated lattice. Then any $\mathcal{L}$-closure system satisfying (19) determines an $\mathcal{L}$-entailment system. 


\subsection{Some conclusions}

The diagram below, not necessarily commutative, gives a taxonomy of interpretations between $\mathcal{L}$-entailment systems $(\mathcal{L}$-ENT), $\mathcal{L}$-institutions $(\mathcal{L}$-INS), and $\mathcal{L}$-closure systems $(\mathcal{L}-$ CLO $)$, that is based on results developed above in the paper.

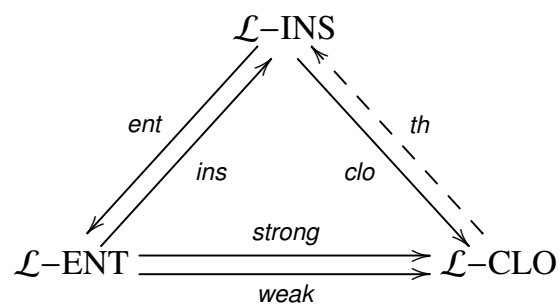

(In this diagram 'ent' is given by Dfn. 4.5 and Prop. 4.1, 'ins' by Prop. 4.2, 'clo' by Prop. 5.5, 'weak' by Prop. 5.3, strong $=$ clo $\circ$ ins by Cor. 5.1, and ' $t h$ ' is the partial mapping given by Fact 5.2.)

We may draw the following conclusions:

1. Both ins and th are embeddings (having ent and clo, respectively, as their retracts) which shows that $\mathcal{L}$ institutions have more structure that $\mathcal{L}$-entailment or $\mathcal{L}$-closure systems. This is to be expected as $\mathcal{L}$-institutions carry an explicit (although fully abstract) semantic component.

2. While the relationship between $\mathcal{L}$-institutions and $\mathcal{L}$-entailment systems is smooth, this is less in the case of the relationship between $\mathcal{L}$-institutions and $\mathcal{L}$-closure systems because of the partiality of the embedding th which is conditioned by the absence of signature morphisms. This situation hints at two important conclusions. One is that the extension from a single signature to a multi signature framework is a highly non-trivial move, it is definitely not a matter of mere indexation. The other point is that $\mathcal{L}$-closure systems seem to be, as mathematical structure, less adequate to model abstract graded consequence than $\mathcal{L}$-entailment systems.

3. While $\mathcal{L}$-entailment systems may be interpreted rather smoothly as $\mathcal{L}$-closure systems (via weak and strong closures), the other way around is less so because of the partiality of th. Hence $\mathcal{L}$-closures seem to be slightly higher abstract than $\mathcal{L}$-entailment systems but in the same time less adequate as mathematical concept for modelling graded consequence. The difference between $\mathcal{L}$-entailment and $\mathcal{L}$-closure in the many-valued situation, which contrasts to the identity between these concepts in the classical two-valued framework, gives another perspective about many-valued truth being much more refined than two-valued truth.

4. In [15] it is argued that consequence operators of [34] and graded consequence relations of [4], i.e. discrete $\mathcal{L}$-closure systems and $\mathcal{L}$-entailment systems in our terminology, respectively, are quite different concepts, the consequence concept proposed by [34] being in reality a crisp one. However the taxonomy above may be interpreted as partially invalidating those claims which in our opinion are too strong. Although different concepts, especially in the discrete situation considered by [15], there are several mutual interpretations between the two concepts (strong and weak on the one hand, and ent $\circ$ th on the other hand).

For the rest of the paper we will work only with $\mathcal{L}$-institutions (for the semantics level) and with $\mathcal{L}$-entailment systems (for the consequence or proof-theoretic level).

\section{Internal versus external logic}

The structure of this section is as follows:

1. We introduce concepts of proof-theoretic logical connectors and quantifiers at the level of abstract $\mathcal{L}$-entailment systems.

2. We introduce concepts of model-theoretic logical connectors and quantifiers at the level of abstract $\mathcal{L}$-institutions We also provide conditions on $\mathcal{L}$ such that these determine the existence of proof-theoretic logical connectors and quantifiers at the level of the semantic entailment system of $\mathcal{L}$-institutions. 


\subsection{Internal logic in $\mathcal{L}$-entailment systems}

The following definition generalizes corresponding proof-theoretic concepts from the classical two-valued institution theory $[9,10,32]$ to many-valued truth.

\section{Definition 6.1. An $\mathcal{L}$-entailment system}

- has conjunction when for any sentences $\rho_{1}$ and $\rho_{2}$ there exists a sentence $\rho^{\prime}$ such that for any set of sentences E,

$$
E \vdash \rho^{\prime}=\left(E \vdash \rho_{1}\right) \wedge\left(E \vdash \rho_{2}\right) ;
$$

- has implication when for any sentences $\rho_{1}$ and $\rho_{2}$ there exists a sentence $\rho^{\prime}$ such that for any set of sentences $E$,

$$
E \vdash \rho^{\prime}=E \cup\left\{\rho_{1}\right\} \vdash \rho_{2} ;
$$

- has disjunction when $\mathcal{L}$ is lattice and for any sentences $\rho_{1}$ and $\rho_{2}$ there exists a sentence $\rho^{\prime}$ such that for any set of sentences $E$,

$$
E \vdash \rho^{\prime}=\left(E \vdash \rho_{1}\right) \vee\left(E \vdash \rho_{2}\right) ;
$$

- has negation when for any sentence $\rho$ there exists a sentence $\rho^{\prime}$ such that for any sentence $e$,

$$
\left\{\rho, \rho^{\prime}\right\} \vdash e=\mathrm{T} ;
$$

- has universal $\chi$-quantification for $\chi: \Sigma \rightarrow \Sigma^{\prime}$ signature morphism when for any $\Sigma^{\prime}$-sentence $\rho^{\prime}$ there exists a $\Sigma$-sentence $\rho$ such that for any set of $\Sigma$-sentences $E$

$$
E \vdash_{\Sigma} \rho=\chi(E) \vdash_{\Sigma^{\prime}} \rho^{\prime} ;
$$

- has existential $\chi$-quantification for $\chi: \Sigma \rightarrow \Sigma^{\prime}$ signature morphism when for any $\Sigma^{\prime}$-sentence $\rho^{\prime}$ there exists a $\Sigma$-sentence $\rho$ such that for any $\Sigma$-sentence $e$

$$
\rho \vdash_{\Sigma} e=\rho^{\prime} \vdash_{\Sigma^{\prime}} \chi(e) .
$$

In two-valued logic the inequalities included in the equation standing for implication are known as the Modus Ponens and the Deduction Theorem. The treatment of quantification by signature morphisms is a traditional feature of institution theory $[8,10,38]$. Note that in concrete situations this allows for first order quantifications when $\chi$ is the extension of $\Sigma$ with first order variables, but also second or even higher order when $\chi$ extends with other operation, relations, or higher order types. Basically, this approach supports quantifications as high as the respective concept of signature supports. In classical two-valued first order logic the equation standing for $\chi$-universal quantification is often known as Generalization Theorem/Rule.

Proposition 6.1. Let $\mathcal{L}$ be a complete meet semi-lattice with $*$ an increasing monotonic binary operation. Let $\left(r^{i}\right)_{i \in I}$ be a family of $\mathcal{L}$-entailment systems that share a common logic syntax. If for each $i \in I, r_{i}$ has conjunction, implication, negation, $\chi$-universal/existential quantification, respectively, then the greatest lower bound $\vdash$ of $\left(\vdash^{i}\right)_{i \in I}$ has conjunction, implication, negation, $\chi$-universal/existential quantification, respectively.

Proof. By Prop. 3.1 the greatest lower bound of any family of $\mathcal{L}$-entailment systems is an $\mathcal{L}$-entailment system too. It remains to show that the respective property of having a certain logical connective or quantification is preserved in the greatest lower bound. However in all cases this consists of a simple straightforward verification that we are going to skip here.

Note that disjunction is missing from the list of logical connectors that are preserved under intersections of $\mathcal{L}$ entailment systems. 
Example 6.1. Within the framework of Ex. 3.3 there is no way to prove $E \vdash^{\prime}(\forall z)(s s z)+(s 0)>(s s s z)$, its truth value being 0 . The rules system employed there, namely that of Ex. 3.1, is too weak for derivation of quantified sentences. The solution is to enhance the respective $\mathcal{L}$-entailment with universal quantification as follows.

Let $r^{\prime \prime}$ be the least $\mathcal{L}$-entailment system greater than $r^{\prime}$ and which has $\chi$-universal quantification for all signature extensions $\chi$ with finite sets of first order variables; its existence is given by Prop. 6.1. Now let us add $z$ as new constant to the signature $\Sigma$ of Ex. 3.3 and denote the resulting signature by $\Sigma(z)$. By the same steps as in the proof of Ex. 3.3 we arrive at

$$
E \vdash_{\Sigma(z)}^{\prime}(s s z)+(s 0)>(s s s z) \geq \frac{1}{n}
$$

Since $r^{\prime} \leq r^{\prime \prime}$ we get

$$
E r_{\Sigma(z)}^{\prime \prime}(s s z)+(s 0)>(s s s z) \geq \frac{1}{n} .
$$

By the universal quantification property of $r^{\prime \prime}$ we get that

$$
E \vdash_{\Sigma}^{\prime \prime}(\forall z)(s s z)+(s 0)>(s s s z) \geq \frac{1}{n}
$$

\subsection{External logic in $\mathcal{L}$-institutions}

The terminology of the following definition is consistent with terminology from [32] $]^{1}$ It generalizes the corresponding model-theoretic concepts from the classical two-valued institution theory $[8,10,32,38]$ to many-valued truth.

\section{Definition 6.2. For any partial order $\mathcal{L}$, an $\mathcal{L}$-institution}

- has external conjunctions when $\mathcal{L}$ has conjunctions and for each $\Sigma$-sentences $\rho_{1}$ and $\rho_{2}$ there exists a $\Sigma$-sentence $\rho_{1} \wedge \rho_{2}$ such that for each $\Sigma$-model $M$,

$$
\left(M \vDash \rho_{1} \wedge \rho_{2}\right)=\left(M \vDash \rho_{1}\right) \wedge\left(M \vDash \rho_{2}\right) ;
$$

- has external implications when $\mathcal{L}$ is a residuated lattice and for any $\Sigma$-sentences $\rho_{1}$ and $\rho_{2}$ there exists a $\Sigma$ sentence $\rho_{1} \Rightarrow \rho_{2}$ such that for each $\Sigma$-model $M$,

$$
\left(M \vDash \rho_{1} \Rightarrow \rho_{2}\right)=\left(M \vDash \rho_{1}\right) \Rightarrow\left(M \vDash \rho_{2}\right) ;
$$

- has external disjunctions when $\mathcal{L}$ has disjunctions and for any $\Sigma$-sentences $\rho_{1}$ and $\rho_{2}$ there exists a $\Sigma$-sentence $\rho_{1} \vee \rho_{2}$ such that for each $\Sigma$-model $M$,

$$
\left(M \vDash \rho_{1} \vee \rho_{2}\right)=\left(M \vDash \rho_{1}\right) \vee\left(M \vDash \rho_{2}\right) ;
$$

- has external negations when $\mathcal{L}$ is a residuated lattice and for each $\Sigma$-sentence $\rho$ there exists a $\Sigma$-sentence $\neg \rho$ such that for each $\Sigma$-model $M$,

$$
(M \vDash \neg \rho)=(M \vDash \rho) \Rightarrow \perp
$$

- has external universal $\chi$-quantification for $\chi: \Sigma \rightarrow \Sigma^{\prime}$ signature morphism when $\mathcal{L}$ is a complete meetsemilattice and for each $\Sigma^{\prime}$-sentence $\rho^{\prime}$ there exists a $\Sigma$-sentence $(\forall \chi) \rho^{\prime}$ such that for any $\Sigma$-model $M$

$$
\left(M \vDash_{\Sigma}(\forall \chi) \rho^{\prime}\right)=\bigwedge\left\{M^{\prime} \vDash_{\Sigma^{\prime}} \rho^{\prime} \mid \operatorname{Mod}(\chi)\left(M^{\prime}\right)=M\right\}
$$

- has external existential $\chi$-quantification for $\chi: \Sigma \rightarrow \Sigma^{\prime}$ signature morphism when $\mathcal{L}$ is a complete joinsemilattice and for each $\Sigma^{\prime}$-sentence $\rho^{\prime}$ there exists a $\Sigma$-sentence $(\exists \chi) \rho^{\prime}$ such that for any $\Sigma$-model $M$

$$
\left(M \vDash_{\Sigma}(\exists \chi) \rho^{\prime}\right)=\bigvee\left\{M^{\prime} \vDash_{\Sigma^{\prime}} \rho^{\prime} \mid \operatorname{Mod}(\chi)\left(M^{\prime}\right)=M\right\}
$$

\footnotetext{
${ }^{1}$ What is called 'external' here and in [32] corresponds to what is called 'internal' in [8, 10]. 'Internal' suggests that the properties characterising the connectives/quantifications are defined as properties of the respective entailment system, while 'external' suggests the involvement of the models as an entity which is outside to the entailment system. One may be tempted to use 'semantic' instead of 'external' and 'syntactic' of 'proof theoretic' instead of 'internal', but this would not be a good choice of terminology because the 'syntactic' stuff for the semantic entailment would have a semantic essence.
} 
Example 6.2. The following properties follow directly from the definition of satisfaction relation in the respective logics captured as $\mathcal{L}$-institutions. Propositional (Ex. 4.1) and first order (Ex. 4.1) many-valued logics and fuzzy multi-algebra (Ex. 4.5) have all external logical connectives. First order and Horn (Ex. 4.3) many-valued logics and fuzzy multi-algebra have external $\chi$-universal quantification for all extensions $\chi$ with first order variables. First order many-valued logic and fuzzy multi-algebra have external $\chi$-existential quantification for all extensions $\chi$ with first order variables. Temporal logic of Ex. 4.4 has external conjunctions and disjunctions when satisfaction is defined by (13) and has external conjunctions when satisfaction is defined by (14). In the latter case, interestingly, the external conjunction of $\rho_{1}$ and $\rho_{2}$ is just $\rho_{1} \vee \rho_{2}$.

Definition 6.3 (Internal semantic connectors/quantifications). We say that an $\mathcal{L}$-institution has an internal connector/quantification when its semantic entailment system has the respective external connector/quantification.

Proposition 6.2. Let $\mathcal{L}$ be a complete residuated lattice and let $\mathcal{I}$ be an $\mathcal{L}$-institution. Then

1. I has internal conjunctions when $\mathcal{I}$ has external conjunctions;

2. I has internal implications when $\mathcal{L}$ is Heyting algebra and $\mathcal{I}$ has external implications;

3. I has internal disjunctions when $\mathcal{L}$ is a Boolean algebra and $\mathcal{I}$ has external disjunctions;

4. I has internal negations when $\mathcal{L}$ is a Heyting algebra and $\mathcal{I}$ has external negations;

5. I has internal universal $\chi$-quantifications when $I$ has external universal $\chi$-quantifications; and

6. I has internal existential $\chi$-quantifications when $I$ has external existential $\chi$-quantifications.

Proof. 1. Assume that $\mathcal{I}$ has external conjunctions. Then

$$
\begin{aligned}
E \vDash \rho_{1} \wedge \rho_{2} & =\bigwedge_{M}\left((M \vDash E) \Rightarrow\left(M \models \rho_{1} \wedge \rho_{2}\right)\right) & & \text { (by Dfn. 4.5) } \\
& =\bigwedge_{M}\left((M \vDash E) \Rightarrow\left(M \models \rho_{1}\right) \wedge\left(M \models \rho_{2}\right)\right) & & \text { (by Dfn. 6.2) } \\
& =\bigwedge_{M}\left(\left((M \vDash E) \Rightarrow\left(M \models \rho_{1}\right)\right) \wedge\left((M \models E) \Rightarrow\left(M \models \rho_{2}\right)\right)\right) & & \text { (by (4) of Prop. } \\
& =\left(E \vDash \rho_{1}\right) \wedge\left(E \models \rho_{2}\right) & & \text { (by Dfn. 4.5). }
\end{aligned}
$$

2. Assume that $\mathcal{L}$ is Heyting algebra and that $\mathcal{I}$ has external implications. Then

$$
\begin{aligned}
E \vDash \rho_{1} \Rightarrow \rho_{2} & =\bigwedge_{M}\left((M \models E) \Rightarrow\left(M \models \rho_{1} \Rightarrow \rho_{2}\right)\right) & & \text { (by Dfn. 4.5) } \\
& =\bigwedge_{M}\left((M \models E) \Rightarrow\left(\left(M \models \rho_{1}\right) \Rightarrow\left(M \models \rho_{2}\right)\right)\right) & & \text { (by Dfn. 6.2) } \\
& =\bigwedge_{M}\left(\left((M \models E) \wedge\left(M \models \rho_{1}\right)\right) \Rightarrow\left(M \models \rho_{2}\right)\right) & & \text { (by (9) of Prop. 2.2) } \\
& =\bigwedge_{M}\left(\left(M \models E \cup\left\{\rho_{1}\right\}\right) \Rightarrow\left(M \models \rho_{2}\right)\right) & & \text { (by Dfn. 4.4) } \\
& =E \cup\left\{\rho_{1}\right\} \vDash \rho_{2} & & \text { (by Dfn. 4.5). }
\end{aligned}
$$

3. Assume that $\mathcal{L}$ is Boolean algebra and that $\mathcal{I}$ has external disjunctions. Then

$$
\begin{aligned}
E \vDash \rho_{1} \vee \rho_{2} & =\bigwedge_{M}\left((M \models E) \Rightarrow\left(M \models \rho_{1} \vee \rho_{2}\right)\right) \\
& =\bigwedge_{M}\left((M \models E) \Rightarrow\left(\left(M \vDash \rho_{1}\right) \vee\left(M \models \rho_{2}\right)\right)\right) \\
& =\bigwedge_{M}\left((M \models E) \Rightarrow\left(M \models \rho_{1}\right)\right) \vee \bigwedge_{M}\left((M \models E) \Rightarrow\left(M \models \rho_{2}\right)\right) \\
& =\left(E \vDash \rho_{1}\right) \vee\left(E \models \rho_{2}\right)
\end{aligned}
$$

4. Assume that $\mathcal{L}$ is Heyting algebra and that $\mathcal{I}$ has external negations. Then

$$
\begin{aligned}
\{\rho, \neg \rho\} \vDash E & =\bigwedge_{M}(M \models\{\rho, \neg \rho\} \Rightarrow M \models E) \\
& =\bigwedge_{M}(((M \vDash \rho) \wedge(M \vDash \neg \rho)) \Rightarrow M \vDash E) \\
& =\bigwedge_{M}(((M \vDash \rho) \wedge((M \models \rho) \Rightarrow \perp)) \Rightarrow M \models E) \\
& =\bigwedge_{M}(\perp \Rightarrow M \models E) \\
& =\top
\end{aligned}
$$

5. Assume that $\mathcal{I}$ has external universal $\chi$-quantifications for $\chi: \Sigma \rightarrow \Sigma^{\prime}$ signature morphism. By Dfn. 4.5, Dfn. 6.2 and by (4) of Prop. 2.1 we have that

$$
E \vDash_{\Sigma}(\forall \chi) \rho^{\prime}=\bigwedge_{M}\left(\bigwedge\left\{M \vDash E \Rightarrow N^{\prime} \vDash \rho^{\prime} \mid \operatorname{Mod}(\chi)\left(N^{\prime}\right)=M\right\}\right) .
$$


By Dfn. 4.5 and by Cor. 4.1 we have that

$$
\chi(E) \models_{\Sigma^{\prime}} \rho^{\prime}=\bigwedge_{M^{\prime}}\left(\operatorname{Mod}(\chi)\left(M^{\prime}\right) \vDash E \Rightarrow M^{\prime} \vDash \rho^{\prime}\right) .
$$

That

$$
\bigwedge_{M}\left(\bigwedge\left\{M \vDash E \Rightarrow N^{\prime} \vDash \rho^{\prime} \mid \operatorname{Mod}(\chi)\left(N^{\prime}\right)=M\right\}\right) \leq \bigwedge_{M^{\prime}}\left(\operatorname{Mod}(\chi)\left(M^{\prime}\right) \vDash E \Rightarrow M^{\prime} \vDash \rho^{\prime}\right) .
$$

follows from the fact that for each $\Sigma^{\prime}$-model $M^{\prime}$

$$
\bigwedge\left\{\operatorname{Mod}(\chi)\left(M^{\prime}\right) \vDash E \Rightarrow N^{\prime} \vDash \rho^{\prime} \mid \operatorname{Mod}(\chi)\left(N^{\prime}\right)=\operatorname{Mod}(\chi)\left(M^{\prime}\right)\right\} \leq\left(\operatorname{Mod}(\chi)\left(M^{\prime}\right) \vDash E \Rightarrow M^{\prime} \vDash \rho^{\prime}\right) .
$$

That

$$
\bigwedge_{M^{\prime}}\left(\operatorname{Mod}(\chi)\left(M^{\prime}\right) \vDash E \Rightarrow M^{\prime} \vDash \rho^{\prime}\right) \leq \bigwedge_{M}\left(\bigwedge\left\{M \vDash E \Rightarrow N^{\prime} \vDash \rho^{\prime} \mid \operatorname{Mod}(\chi)\left(N^{\prime}\right)=M\right\}\right)
$$

follows from the fact that for each $\Sigma$-model $M$ and for each $\Sigma^{\prime}$-model $N^{\prime}$ such that $M=\operatorname{Mod}(\chi)\left(N^{\prime}\right)$,

$$
\bigwedge_{M^{\prime}}\left(\operatorname{Mod}(\chi)\left(M^{\prime}\right) \vDash E \Rightarrow M^{\prime} \vDash \rho^{\prime}\right) \leq\left(M \vDash E \Rightarrow N^{\prime} \vDash \rho^{\prime}\right) .
$$

6. Assume that $\mathcal{I}$ has external existential $\chi$-quantifications for $\chi: \Sigma \rightarrow \Sigma^{\prime}$ signature morphism. By Dfn. 4.5 and Dfn. 6.2 we have that

$$
(\exists \chi) \rho^{\prime} \vDash_{\Sigma} e=\bigwedge_{M}\left(\bigvee\left\{N^{\prime} \vDash_{\Sigma^{\prime}} \rho^{\prime} \mid \operatorname{Mod}(\chi)\left(N^{\prime}\right)=M\right\} \Rightarrow\left(M \vDash_{\Sigma} e\right)\right) .
$$

By Dfn. 4.5 and by Cor. 4.1 we have that

$$
\rho^{\prime} \vDash_{\Sigma^{\prime}} \chi(e)=\bigwedge_{M^{\prime}}\left(M^{\prime} \vDash_{\Sigma^{\prime}} \rho^{\prime} \Rightarrow \operatorname{Mod}(\chi)\left(M^{\prime}\right) \vDash_{\Sigma} e\right)
$$

That

$$
\bigwedge_{M}\left(\bigvee\left\{N^{\prime} \vDash_{\Sigma^{\prime}} \rho^{\prime} \mid \operatorname{Mod}(\chi)\left(N^{\prime}\right)=M\right\} \Rightarrow\left(M \vDash_{\Sigma} e\right)\right) \leq \bigwedge_{M^{\prime}}\left(M^{\prime} \vDash_{\Sigma^{\prime}} \rho^{\prime} \Rightarrow \operatorname{Mod}(\chi)\left(M^{\prime}\right) \vDash_{\Sigma} e\right)
$$

follows from the fact that for each $\Sigma^{\prime}$-model $M^{\prime}$, by (2) of Prop. 2.1

$$
\bigvee\left\{N^{\prime} \vDash_{\Sigma^{\prime}} \rho^{\prime} \mid \operatorname{Mod}(\chi)\left(N^{\prime}\right)=\operatorname{Mod}(\chi)\left(M^{\prime}\right)\right\} \Rightarrow\left(\operatorname{Mod}(\chi)\left(M^{\prime}\right) \vDash_{\Sigma} e\right) \leq\left(M^{\prime} \vDash_{\Sigma^{\prime}} \rho^{\prime} \Rightarrow \operatorname{Mod}(\chi)\left(M^{\prime}\right) \vDash_{\Sigma} e\right)
$$

That

$$
\bigwedge_{M^{\prime}}\left(M^{\prime} \vDash_{\Sigma^{\prime}} \rho^{\prime} \Rightarrow \operatorname{Mod}(\chi)\left(M^{\prime}\right) \vDash_{\Sigma} e\right) \leq \bigwedge_{M}\left(\bigvee\left\{N^{\prime} \vDash_{\Sigma^{\prime}} \rho^{\prime} \mid \operatorname{Mod}(\chi)\left(N^{\prime}\right)=M\right\} \Rightarrow\left(M \vDash_{\Sigma} e\right)\right)
$$

follows from the fact that for each $\Sigma$-model $M$

$$
\bigwedge_{M^{\prime}}\left(M^{\prime} \vDash_{\Sigma^{\prime}} \rho^{\prime} \Rightarrow \operatorname{Mod}(\chi)\left(M^{\prime}\right) \vDash_{\Sigma} e\right) \leq \bigvee\left\{N^{\prime} \vDash_{\Sigma^{\prime}} \rho^{\prime} \mid \operatorname{Mod}(\chi)\left(N^{\prime}\right)=M\right\} \Rightarrow\left(M \vDash_{\Sigma} e\right)
$$

which is shown as follows. For each $N^{\prime} \operatorname{such}$ that $\operatorname{Mod}(\chi)\left(N^{\prime}\right)=M$ we have that

$$
\bigwedge_{M^{\prime}}\left(M^{\prime} \vDash_{\Sigma^{\prime}} \rho^{\prime} \Rightarrow \operatorname{Mod}(\chi)\left(M^{\prime}\right) \vDash_{\Sigma} e\right) \leq\left(N^{\prime} \vDash_{\Sigma^{\prime}} \rho^{\prime}\right) \Rightarrow\left(M \vDash_{\Sigma} e\right)
$$

hence

$$
\left(\bigwedge_{M^{\prime}}\left(M^{\prime} \vDash_{\Sigma^{\prime}} \rho^{\prime} \Rightarrow \operatorname{Mod}(\chi)\left(M^{\prime}\right) \vDash_{\Sigma} e\right)\right) *\left(N^{\prime} \vDash_{\Sigma^{\prime}} \rho^{\prime}\right) \leq\left(M \vDash_{\Sigma} e\right) .
$$

It follows that

$$
\bigvee\left\{\left(\bigwedge_{M^{\prime}}\left(M^{\prime} \vDash_{\Sigma^{\prime}} \rho^{\prime} \Rightarrow \operatorname{Mod}(\chi)\left(M^{\prime}\right) \vDash_{\Sigma} e\right)\right) *\left(N^{\prime} \vDash_{\Sigma^{\prime}} \rho^{\prime}\right) \mid \operatorname{Mod}(\chi)\left(N^{\prime}\right)=M\right\} \leq\left(M \vDash_{\Sigma} e\right)
$$

By (6) of Prop. 2.1 it follows that

$$
\bigwedge_{M^{\prime}}\left(M^{\prime} \vDash_{\Sigma^{\prime}} \rho^{\prime} \Rightarrow \operatorname{Mod}(\chi)\left(M^{\prime}\right) \vDash_{\Sigma} e\right) * \bigvee\left\{N^{\prime} \vDash_{\Sigma^{\prime}} \rho^{\prime} \mid \operatorname{Mod}(\chi)\left(N^{\prime}\right)=M\right\} \leq\left(M \vDash_{\Sigma} e\right)
$$

hence (20).

One immediate application of Prop. 6.2 is the following soundness result. 
Corollary 6.1. Let $\mathcal{I}$ be an $\mathcal{L}$-institution that has external conjunction, implication, negation, or $\chi$-universal/existential quantification, respectively. In the case of implication or negation let us also assume that $\mathcal{L}$ is a Heyting algebra. Let $\vdash$ be an $\mathcal{L}$-entailment system that is sound for $\mathcal{I}$. Then the least $\mathcal{L}$-entailment system $r^{\prime}$ having conjunction, implication, negation, or $\chi$-universal/existential quantification, respectively, and greater than $\vdash$ is sound for $\mathcal{I}$ too.

Proof. We consider the semantic $\mathcal{L}$-entailment system of $\mathcal{I}$ (as given by Prop. 4.1). According to Prop. 6.2 this has the respective internal logic property. Since by the soundness assumption $r \leq \models$, it follows that $r^{\prime} \leq \models$.

Example 6.3. In the case of the $\mathcal{L}$-entailment system $r^{\prime \prime}$ of Ex. 6.1 by the conclusion of Ex. 4.7 we know that $r^{\prime}$ is sound for the $\mathcal{L}$-institution defined in Ex. 4.7. By the definition of the satisfaction relation in that $\mathcal{L}$-institution, which is like in the institution of first order many-valued logic (Ex. 4.2), it is clear that the respective $\mathcal{L}$-institution has external $\chi$-universal quantifications for any signature extension $\chi$ with a finite set of variables. By Cor. 6.1 it follows that $r^{\prime \prime}$ is sound, hence from Ex. 6.1 we may further deduce that

$$
E \models_{\Sigma}(\forall z)(s s z)+(s 0)>(s s s z) \geq \frac{1}{n} .
$$

\section{Graded compactness}

In this section we first introduce a concept of compactness for $\mathcal{L}$-entailment, then provide sufficient conditions such that finitary $\mathcal{L}$-rules systems generate compact $\mathcal{L}$-entailment systems. We also show that compactness is preserved by the logical connectors and quantifications. At the end of this section we introduce a model-theoretic concept of compactness for $\mathcal{L}$-institutions.

\subsection{Compact entailment}

Definition 7.1 (Compact entailment). An $\mathcal{L}$-entailment system (Sign, Sen, $\left.\left(\vdash_{\Sigma}\right)_{\Sigma \in \mid \text { Sign } \mid}\right)$ is compact when for any entailment $E r_{\Sigma} \rho$ we have

$$
E \vdash \gamma=\bigvee\left\{E_{0} \vdash \gamma \mid E_{0} \text { finite } \subseteq E\right\}
$$

Proposition 7.1. In any compact $\mathcal{L}$-entailment system (Sign, Sen, $\left.\left(\vdash_{\Sigma}\right)_{\Sigma \in|\operatorname{Sign}|}\right)$ such that the meet operation $\wedge$ is joincontinuous, for any compact $k \in L$, if $k \leq(E \vdash \gamma)$ then there exists finite $E_{0} \subseteq E$ such that $k \leq\left(E_{0} \vdash \gamma\right)$.

Proof. Let $k \in L$ be a compact element and $E \vdash \gamma$. We thus have the following:

$$
\begin{aligned}
k=k \wedge(E \vdash \gamma) & =k \wedge \bigvee\left\{E_{0} \vdash \gamma \mid E_{0} \subseteq E \text { finite }\right\} \\
& \left.=\bigvee\left\{k \wedge\left(E_{0} \vdash \gamma\right) \mid E_{0} \subseteq E \text { finite }\right\} \quad \text { (by the continuity of } \wedge\right) .
\end{aligned}
$$

The set $\left\{k \wedge\left(E_{0} \vdash \gamma\right) \mid E_{0} \subseteq E\right.$ finite $\}$ is directed since for any finite $E_{0}, E_{0}^{\prime} \subseteq E$ by Monotonicity we have that $\left(E_{0} \vdash \gamma\right),\left(E_{0}^{\prime} \vdash \gamma\right) \leq\left(E_{0} \cup E_{0}^{\prime} \vdash \gamma\right)$. Hence by the compactness of $k$ there exists finite $E_{0} \subseteq E$ such that $k=k \wedge\left(E_{0} \vdash \gamma\right)$, which means $k \leq\left(E_{0} \vdash \gamma\right)$.

Note that the classical concept of compactness for entailment systems (e.g. [30]) is covered by Dfn. 7.1 when we let $\mathcal{L}$ be the two-valued Boolean algebra $\{\perp, T\}$ and consider $k=\top$. Note that in this situation the other case, namely $k=\perp$ is trivial.

Proposition 7.2. Let (Sign, Sen, $\left.\left(\vdash_{\Sigma}\right)_{\Sigma \in|\operatorname{Sign}|}\right)$ be a $\mathcal{L}$-entailment system such that $\mathcal{L}$ is a complete lattice with a joincontinuous binary operation $*$ and such that the meet operation $\wedge$ is join-continuous too. Then

$$
E \vdash^{\omega} \gamma=\bigvee\left\{E_{0} \vdash \gamma \mid E_{0} \text { finite } \subseteq E\right\}
$$

defines an $\mathcal{L}$-entailment system over the logic syntax (Sign, Sen).

Moreover if $\vdash$ has conjunctions, implications, disjunctions, negations, or $\chi$-universal/existential quantifications, then $\vdash^{\omega}$ has them also. 
Proof. We have to show that $r^{\omega}$ satisfies the axioms of Dfn. 3.4.

(Reflexivity) This is trivial.

(Monotonicity) Let $E \subseteq E^{\prime} \subseteq \operatorname{Sen}(\Sigma)$. Then

$$
\begin{aligned}
E \vdash^{\omega} \gamma & =\bigvee\left\{E_{0} \vdash \gamma \mid E_{0} \text { finite } \subseteq E\right\} & & \left(\text { by definition of } \vdash^{\omega}\right) \\
& \leq \bigvee\left\{E_{0} \vdash \gamma \mid E_{0} \text { finite } \subseteq E^{\prime}\right\} & & \left(\text { since } E \subseteq E^{\prime}\right) \\
& =E^{\prime} \vdash^{\omega} \gamma & &
\end{aligned}
$$

(Transitivity) Let $E, \Gamma \subseteq \operatorname{Sen}(\Sigma)$ and $\gamma^{\prime} \in \operatorname{Sen}(\Sigma)$. Then

$$
\begin{aligned}
\left(E \vdash^{\omega} \Gamma\right) *\left(\Gamma \vdash^{\omega} \gamma^{\prime}\right)= & \left(\bigwedge_{\gamma \in \Gamma}\left(E \vdash^{\omega} \gamma\right)\right) *\left(\Gamma \vdash^{\omega} \gamma^{\prime}\right) \\
= & \left(\bigwedge_{\gamma \in \Gamma}\left(\bigvee\left\{E_{0} \vdash \gamma \mid E_{0} \text { finite } \subseteq E\right\}\right)\right) *\left(\bigvee\left\{\Gamma_{0} \vdash \gamma^{\prime} \mid \Gamma_{0} \text { finite } \subseteq \Gamma\right\}\right) \\
& \left(\text { by definition of } \vdash^{\omega}\right) \\
= & \bigvee_{\Gamma_{0}}\left(\left(\bigwedge_{\gamma \in \Gamma}\left(\bigvee\left\{E_{0} \vdash \gamma \mid E_{0} \text { finite } \subseteq E\right\}\right)\right) *\left(\Gamma_{0} \vdash \gamma^{\prime}\right)\right) \\
& (\text { by the continuity of } *) \\
\leq & \bigvee_{\Gamma_{0}}\left(\left(\bigwedge_{\gamma \in \Gamma_{0}}\left(\bigvee\left\{E_{0} \vdash \gamma \mid E_{0} \text { finite } \subseteq E\right\}\right)\right) *\left(\Gamma_{0} \vdash \gamma^{\prime}\right)\right) \\
& (\text { by continuity, * is increasing monotonic) } \\
= & \bigvee_{\Gamma_{0}}\left(\left(\bigvee\left\{\bigwedge_{\gamma \in \Gamma_{0}}\left(E_{0} \vdash \gamma\right) \mid E_{0} \text { finite } \subseteq E\right\}\right) *\left(\Gamma_{0} \vdash \gamma^{\prime}\right)\right) \\
& \left(\text { by the continuity of } \wedge \text { since } \Gamma_{0} \text { is finite }\right) \\
= & \bigvee_{\Gamma_{0}}\left(\left(\bigvee\left\{E_{0} \vdash \Gamma_{0} \mid E_{0} \text { finite } \subseteq E\right\}\right) *\left(\Gamma_{0} \vdash \gamma^{\prime}\right)\right) \\
= & \left.\bigvee_{\Gamma_{0}} \bigvee_{E_{0}}\left(\left(E_{0} \vdash \Gamma_{0}\right) *\left(\Gamma_{0} \vdash \gamma^{\prime}\right)\right) \text { (by the continuity of } *\right) \\
\leq & \bigvee_{\Gamma_{0}} \bigvee_{E_{0}}\left(E_{0} \vdash \gamma^{\prime}\right)(\text { by Transitivity for } \vdash) \\
= & \left.\bigvee_{E_{0}}\left(E_{0} \vdash \gamma^{\prime}\right)=\left(E \vdash^{\omega} \gamma^{\prime}\right) \text { (by definition of } \vdash^{\omega}\right) .
\end{aligned}
$$

(Translation) Let $E \subseteq \operatorname{Sen}(\Sigma), \gamma \in \operatorname{Sen}(\Sigma)$ and $\varphi: \Sigma \rightarrow \Sigma^{\prime} \in \operatorname{Sign}$. Then

$$
\begin{aligned}
E \vdash^{\omega} \gamma & =\bigvee\left\{E_{0} \vdash \gamma \mid E_{0} \text { finite } \subseteq E\right\} & & \text { (by definition of } \vdash^{\omega} \text { ) } \\
& \leq \bigvee\left\{\varphi\left(E_{0}\right) \vdash \varphi(\gamma) \mid E_{0} \text { finite } \subseteq E\right\} & & \text { (by Translation for } \vdash \text { ) } \\
& =\bigvee\left\{E_{0}^{\prime} \vdash \varphi(\gamma) \mid E_{0}^{\prime} \text { finite } \subseteq \varphi(E)\right\} & & \\
& =\varphi(E) \vdash^{\omega} \varphi(\gamma) & & \text { (by definition of } \left.\vdash^{\omega}\right) .
\end{aligned}
$$

The second part of this proposition follows by straightforward calculations that we skip here.

Note that in Prop. 7.2, according to (6) of Prop. 2.1, the join-continuity of $*$ is guaranteed whenever $\mathcal{L}$ is a residuated lattice and $*$ is its monoidal operation. Moreover when $\mathcal{L}$ is Heyting algebra the join-continuity of $\wedge$ also holds.

Corollary 7.1. If $\mathcal{L}$ is a complete residuated lattice such that $\wedge$ is join-continuous then any finitary $\mathcal{L}$-rules system generates a compact $\mathcal{L}$-entailment system.

Proof. Let $r$ be a finitary $\mathcal{L}$-rules system and let $r^{\prime}$ be the entailment system generated by $r$. Note that the condition of continuity for $*$ in Prop. 7.2 comes as a consequence of $\mathcal{L}$ being residuated lattice (6) of Prop. 2.1. Let ${r^{\prime}}^{\omega}$ be the entailment system determined by ${r^{\prime}}^{\prime}$ according to Prop. 7.2. We have to show that for any $E, \gamma,\left(E \vdash^{\prime} \gamma\right)=\left(E r^{\omega} \gamma\right)$. By Monotonicity it is clear that $\left(E \vdash^{\prime \omega} \gamma\right) \leq\left(E \vdash^{\prime} \gamma\right)$. Since $\vdash^{\prime}$ is the least entailment system greater than $\vdash$, by Prop. 7.2 it is enough to show that for any $E, \gamma,(E \vdash \gamma) \leq\left(E \vdash^{\prime \omega} \gamma\right)$.

For any $E, \gamma$, when $E$ is infinite we trivially have that $(E \vdash \gamma) \leq\left(E \vdash^{\prime \omega} \gamma\right)$. When $E$ is finite from the definition of $\vdash^{\prime \omega}$, by the finiteness of $E$ we have that $\left(E \vdash^{\prime} \gamma\right)=\left(E{\vdash^{\prime}}^{\omega} \gamma\right)$. Hence $(E \vdash \gamma) \leq\left(E \vdash^{\prime \omega} \gamma\right)$.

Cor. 7.1 generalizes a corresponding two-valued result from $[9,10]$ to many-valued truth.

Corollary 7.2. Given a compact $\mathcal{L}$-entailment system $\vdash$, the $\mathcal{L}$-entailment system generated by $\vdash$ and which has conjunction/implication/disjunction/negation and/or $\chi$-universal/existential quantification is compact too.

Example 7.1. According to Cor. 7.1, since the $\mathcal{L}$-rules system $\vdash$ of Ex. 3.3 is obviously finitary, it follows that the $\mathcal{L}$ entailment system $r^{\prime}$ is compact. Since in that example $\mathcal{L}$ is finite, all its elements are compact, hence the conclusion of Cor. 7.1 reads as follows: for each $1 \leq k \leq n$ if $\frac{k}{n} \leq\left(E \vdash^{\prime} \gamma\right)$ then there exists finite $E_{0} \subseteq E$ such that $\frac{k}{n} \leq\left(E_{0} \vdash^{\prime} \gamma\right)$. When $k=n$ this reads as follows: if $\left(E \vdash^{\prime} \gamma\right)=1$ then there exists finite $E_{0} \subseteq E$ such that $\left(E_{0} \vdash^{\prime} \gamma\right)=1$. Moreover, by Cor. 7.2 compactness is extended from $r^{\prime}$ to $\vdash^{\prime \prime}$ of Ex. 6.1 . 


\section{Conclusions and Future Research}

In this paper we have introduced a fully abstract concept of graded multi-signature entailment, we have defined a graded semantic consequence in many-valued institutions, and we showed that the latter satisfies the axioms of the former. Moreover this interpretation of many-valued institutions as graded entailment systems has a left-inverse, a construction that we have used to define a semantic-inspired interpretation of graded entailment systems as closure operators on fuzzy theories. We have also defined a weaker closure on fuzzy theories in graded multi-signature entailment systems. We have seen that in multi-signature frameworks the move in the opposite direction is virtually unfeasible. This situation has led us to argue that closure systems are too abstract for being an effective conceptual tool in the study of graded consequence.

Then we have defined logical connectors and quantifiers both proof theoretically, for graded entailment systems, and semantically, for many-valued institutions. Our institution theoretic treatment of quantifiers is fully abstract and relies upon the multi-signature aspect of our approach. We have studied conditions when semantical logical connectors and quantifiers determine their proof theoretic correspondents for the graded semantic consequence.

In the last section we have defined a concept of compactness for graded entailment, that covers the compactness of classical two-valued entailment systems. We have proved that graded entailment systems that are freely generated by systems of finitary rules are compact, and that compactness is preserved by logical connectors and quantifiers.

In this paper we have also soundness of graded entailment with respect to many-valued institution theoretic semantics and showed that soundness is transferred automatically from rules to entailments and further through logical connectors and quantifiers.

Our study rises a number of open questions from which we mention the following:

1. We have seen that in the classical two-valued framework the weak and strong closures in entailment systems are the same concept, i.e. we have that $X^{\circ}=X^{* *}\left(=X^{\bullet}\right)$. What is a sensible set of sufficient conditions such that this equality holds also in proper many-valued setups?

2. In any $\mathcal{L}$-institution, for any $\Sigma$-theory $X$ and any $\Gamma \subseteq \operatorname{Sen}(\Sigma)$ we let $X \mid \Gamma$ be the $\Sigma$-theory defined by

$$
(X \mid \Gamma) \rho= \begin{cases}X(\rho) & \text { when } \rho \in \Gamma \\ \perp & \text { when } \rho \notin \Gamma .\end{cases}
$$

The following concept represents a generalization of the corresponding concept of model theoretic compactness from classical two-valued institutions $[8,10]$ to many-valued institutions. An $\mathcal{L}$-institution is $m$-compact when each $\Sigma$-theory $X$ is consistent whenever $X \mid \Gamma_{0}$ is consistent for each finite $\Gamma_{0} \subseteq \operatorname{Sen}(\Sigma)$. According to [8, 10], in classical two-valued institutions that have negations and conjunctions (in the sense of Dfn. 6.2) m-compactness is equivalent to the compactness of the semantic consequence $=$. Classical first order logic is such an example. These two conditions do not seem to suffice to ensure this equivalence in proper many-valued contexts. Then what is a sensible set of sufficient conditions for this equivalence to hold? Or, less ambitious, establish a meaningful relationship between m-compactness and the compactness of the graded semantic consequence.

3. Establish relationships between our concept of compactness of graded entailment and Pavelka's styled [34] compactness of the closure operators $\left(_{-}\right)^{\circ}$ and (_- $)^{\bullet}$.

Another further research directions arising from our work include study of properties such as interpolation, definability, etc. in an abstract graded framework.

\section{Acknowledgements}

This work has been supported by a grant of the Romanian National Authority for Scientific Research, CNCSUEFISCDI, project number PN-II-ID-PCE-2011-3-0439. Thanks to Ionuţ Ţuţu for enlightening discussions around the temporal logic example.

\section{References}

[1] Jean-Yves Béziau. 13 questions about universal logic. Bulletin of the Section of Logic, 35(2/3):133-150, 2006

[2] Jean-Yves Béziau, editor. Universal Logic: an Anthology. Studies in Universal Logic. Springer Basel, 2012.

[3] Peter Burmeister. Partial algebra - an introductory survey. Algebra Universalis, 15:306-358, 1982. 
[4] Mihir K. Chakraborty. Use of fuzzy set theory in introducing graded consequence in multiple valued logic. In M.M. Gupta and T. Yamakawa, editors, Fuzzy Logic in Knowledge-Based Systems, Decision and Control, pages 247-257. Elsevier Science Publishers, B.V., North Holland, 1988.

[5] Mihir K. Chakraborty. Graded consequence: further studies. Journal of Applied Non-Classical Logics, 5(2):127-137, 1995.

[6] Petr Cintula and Petr Hájek. On theories and models in fuzzy predicate logic. Journal of Symbolic Logic, 71(3):832-863, 2006.

[7] Răzvan Diaconescu. Grothendieck institutions. Applied Categorical Structures, 10(4):383-402, 2002. Preliminary version appeared as IMAR Preprint 2-2000, ISSN 250-3638, February 2000.

[8] Răzvan Diaconescu. Institution-independent ultraproducts. Fundamenta Informatica, 55(3-4):321-348, 2003.

[9] Răzvan Diaconescu. Proof systems for institutional logic. Journal of Logic and Computation, 16(3):339-357, 2006.

[10] Răzvan Diaconescu. Institution-independent Model Theory. Birkhäuser, 2008.

[11] Răzvan Diaconescu. On quasi-varieties of multiple valued logic models. Mathematical Logic Quarterly, 57(2):194-203, 2011.

[12] Răzvan Diaconescu. Structural induction in institutions. Information and Computation, 209(9):1197-1222, 2011.

[13] Răzvan Diaconescu. Three decades of institution theory. In Jean-Yves Béziau, editor, Universal Logic: an Anthology, pages 309-322. Springer Basel, 2012.

[14] Răzvan Diaconescu. Institutional semantics for many-valued logics. Fuzzy Sets and Systems, 218:32-52, 2013.

[15] Soma Dutta, Sanjukta Basu, and Mihir K. Chakraborty. Many-valued logics, fuzzy logics and graded consequence: A comparative appraisal. In K. Lodaya, editor, ICLA 2013, volume 7750 of Lecture Notes in Artificial Intelligence, pages 197-209, 2013.

[16] Patrick Eklund and Robert Helgesson. Monadic extensions of institutions. Fuzzy Sets and Systems, 161:2354-2368, 2010.

[17] José L. Fiadeiro and José F. Costa. Mirror, mirror in my hand: A duality between specifications and models of process behaviour. Mathematical Structures in Computer Science, 6(4):353-373, 1996.

[18] José L. Fiadeiro and Amilcar Sernadas. Structuring theories on consequence. In Donald Sannella and Andrzej Tarlecki, editors, Recent Trends in Data Type Specification, volume 332 of Lecture Notes in Computer Science, pages 44-72. Springer, 1988.

[19] Nikolaos Galatos, Peter Jipsen, Tomasz Kowalski, and Hiroakira Ono. Residuated Lattices: An Algebraic Glimpse at Substructural Logics. Elsevier, 2007.

[20] Giangiacomo Gerla. Fuzzy Logic: Mathematical Tools for Approximate Reasoning. Kluwer, 2001.

[21] Joseph Goguen. The logic of inexact concepts. Synthese, 19:325-373, 1968.

[22] Joseph Goguen and Rod Burstall. Introducing institutions. In Edward Clarke and Dexter Kozen, editors, Proceedings, Logics of Programming Workshop, volume 164 of Lecture Notes in Computer Science, pages 221-256. Springer, 1984.

[23] Joseph Goguen and Rod Burstall. Institutions: Abstract model theory for specification and programming. Journal of the Association for Computing Machinery, 39(1):95-146, 1992.

[24] George Grátzer. Lattice theory: Foundation. Springer Basel, 2011.

[25] Petr Hájek. Metamathematics of Fuzzy Logic. Kluwer, 1998.

[26] Heinrich Hussmann. Nondeterminism in Algebraic Specifications and Algebraic Program. Birkhaüser, 1993.

[27] Yngve Lamo. The Institution of Multialgebras - a general framework for algebraic software development. PhD thesis, University of Bergen, 2003.

[28] Saunders Mac Lane. Categories for the Working Mathematician. Springer, second edition, 1998.

[29] Brian Mayoh. Galleries and institutions. Technical Report DAIMI PB-191, Aarhus University, 1985.

[30] José Meseguer. General logics. In H.-D. Ebbinghaus et al., editors, Proceedings, Logic Colloquium, 1987, pages 275-329. North-Holland, 1989.

[31] José Meseguer. Membership algebra as a logical framework for equational specification. In F. Parisi-Pressice, editor, Proc. WADT’97, volume 1376 of Lecture Notes in Computer Science, pages 18-61. Springer, 1998.

[32] Till Mossakowski, Joseph Goguen, Răzvan Diaconescu, and Andrzej Tarlecki. What is a logic? In Jean-Yves Béziau, editor, Logica Universalis, pages 113-133. Birkhäuser, 2005.

[33] Hiroakira Ono. Substructural logics and residuated lattices - an introduction. In V. F. Hendricks and J. Malinowski, editors, 50 Years of Studia Logica, volume 20 of Trends in Logic, pages 177-212. Kluwer/Springer, 2003.

[34] Jan Pavelka. On fuzzy logic i - many-valued rules of inference. Zeitscher for Math. Logik und Grundlagen d. Math, 25:45-52, 1979.

[35] Florian Rabe. A framework for combining model and proof theory. Mathematical Structures in Computer Science, 2012. Submitted.

[36] Donald Sannella and Andrzej Tarlecki. Foundations of Algebraic Specifications and Formal Software Development. Springer, 2012.

[37] Dana Scott. Completeness and axiomatizability in many-valued logic. In Leon Henkin et al., editor, Proceedings, Tarski Symposium, pages 411-435. American Mathematical Society, 1974.

[38] Andrzej Tarlecki. Bits and pieces of the theory of institutions. In David Pitt, Samson Abramsky, Axel Poigné, and David Rydeheard, editors, Proceedings, Summer Workshop on Category Theory and Computer Programming, volume 240 of Lecture Notes in Computer Science, pages 334-360. Springer, 1986.

[39] Alfred Tarski. On some fundamental concepts of metamathematics. In Logic, Semantics, Metamathematics, pages 30-37. Oxford University Press, 1956.

[40] George Voutsadakis. Categorical abstract algebraic logic: Algebrizable institutions. Applied Categorical Structues, 10:531-568, 2002.

[41] Michał Walicki. Algebraic Specification of Nondeterminism. PhD thesis, Department of Informatics, University of Bergen, 1993.

[42] Michał Walicki and Sigurd Meldal. Algebraic approaches to nondeterminism - an overview. ACM Computing Surveys, $29,1997$. 